\title{
Regulation of Fasciclin II and Synaptic Terminal Development by the Splicing Factor Beag
}

\author{
Erin S. Beck, Gabriel Gasque, Wendy L. Imlach, Wei Jiao, Ben Jiwon Choi, Pao-Shu Wu, Matthew L. Kraushar, \\ and Brian D. McCabe \\ Center for Motor Neuron Biology and Disease, Department of Pathology and Cell Biology and Department of Neuroscience, Columbia University Medical \\ Center, New York, New York 10032
}

Pre-mRNA alternative splicing is an important mechanism for the generation of synaptic protein diversity, but few factors governing this process have been identified. From a screen for Drosophila mutants with aberrant synaptic development, we identified beag, a mutant with fewer synaptic boutons and decreased neurotransmitter release. Beag encodes a spliceosomal protein similar to splicing factors in humans and Caenorhabditis elegans. We find that both beag mutants and mutants of an interacting gene dsmul have changes in the synaptic levels of specific splice isoforms of Fasciclin II (FasII), the Drosophila ortholog of neural cell adhesion molecule. We show that restoration of one splice isoform of FasII can rescue synaptic morphology in beag mutants while expression of other isoforms cannot. We further demonstrate that this FasII isoform has unique functions in synaptic development independent of transsynaptic adhesion. beag and $d s m u 1$ mutants demonstrate an essential role for these previously uncharacterized splicing factors in the regulation of synapse development and function.

\section{Introduction}

Synapses are exquisitely specialized cell-cell contacts characterized by dense multiprotein complexes surrounding the tightly apposed presynaptic and postsynaptic membranes. Bridging this cleft are synaptic adhesion molecules (SAMs), which provide adhesive structural stability and act as transsynaptic signaling proteins (Dalva et al., 2007). Many SAM genes have extensive alternative splice isoform diversity, with some such as neurexins capable of producing hundreds of different protein splice isoforms (Ullrich et al., 1995).

The neural cell adhesion molecule (NCAM) is an extensively studied homophilic synaptic adhesion molecule (Hartz and Ronn, 2010; Kristiansen and Hortsch, 2010; Muller et al., 2010) that can modulate signaling through its intracellular domain (Ditlevsen and Kolkova, 2010). Fasciclin II (FasII), the Drosophila ortholog of NCAM, is essential for normal synaptic development and growth (Packard et al., 2003; Kristiansen and Hortsch, 2010). In fasII-null mutants initial neuromuscular junction (NMJ) syn-

Received July 19, 2011; revised March 23, 2012; accepted April 4, 2012.

Author contributions: E.S.B. and B.D.M. designed research; E.S.B., G.G., W.L.I., W.J., B.J.C., P.-S.W., M.LK., and B.D.M. performed research; B.D.M. contributed unpublished reagents/analytic tools; E.S.B. and B.D.M. analyzed data; E.S.B. and B.D.M. wrote the paper.

E.S.B. was funded by the National Institutes of Health (NIH) GM07367 and B.D.M. was funded by NIH NS075572. We would like to thank James Manley and Rachel Kraut for advice and members of the McCabe lab for helpful discussions. We thank Chris Henderson, Wesley Grueber, and Jane Dodd for careful review of the manuscript. We are very grateful to Hermann Aberle, Karen Zito, Corey Goodman, Ji-Wu Wang, the Developmental Studies Hybridoma Bank, and the Bloomington stock center for reagents.

The authors declare no competing financial interests.

Correspondence should be addressed to Brian D. McCabe, Center for Motor Neuron Biology and Disease, Department of Pathology and Cell Biology and Department of Neuroscience, Columbia University Medical Center, 630 W. 168th Street, New York, New York 10032. E-mail: brian@mccabelab.org.

DOI:10.1523/JNEUROSCI.3717-11.2012

Copyright $\odot 2012$ the authors $\quad 0270-6474 / 12 / 327058-16 \$ 15.00 / 0$ aptic contacts form but later retract (Schuster et al., 1996a), while in partial loss-of-function mutants, synaptic terminal size is reduced (Stewart et al., 1996). Regulation of synaptic FasII and NCAM is also important for synaptic plasticity (Muller et al., 1996; Schuster et al., 1996b; Cremer et al., 1997; Koh et al., 1999; Sigrist et al., 2003).

Both NCAM and FasII pre-mRNAs undergo alternative splicing to generate multiple protein isoforms (Cunningham et al., 1987). NCAM has three major splice isoforms, NCAM 120, 140, and 180, which have identical extracellular domains. NCAM 120 is attached to the membrane via a glycophosphatidylinositol (GPI) linkage, while NCAM 140 and 180 are transmembrane proteins. The expression pattern and intracellular binding partners differ between NCAM isoforms (Pollerberg et al., 1985, 1986; Persohn et al., 1989). Similarly, Drosophila FasII undergoes alternative splicing to create two transmembrane isoforms and two additional isoforms, one of which may be GPI-linked (Grenningloh et al., 1991; Lin et al., 1994). The distinct functional requirement for these isoforms in synapse development is unclear. Similar to most SAM genes, the factors that regulate alternative splicing of NCAM and FasII are unknown. Proteomic analysis of spliceosomes in vertebrates and Drosophila has identified many proteins with the potential to influence splicing; however, few splicing factors that regulate neuronal protein diversity have been characterized (Neubauer et al., 1998; Zhou et al., 2002; Herold et al., 2009).

Here we identify novel mutants in two genes, beag and dsmu1, that regulate Drosophila synaptic morphology and function. The proteins encoded by these genes are components of the Drosophila spliceosome (Herold et al., 2009) and are related to human and Caenorhabditis elegans spliceosomal proteins (Neubauer et al., 1998; Assier et al., 1999). We show that the aberrant synaptic morphology 
of beag mutants is due to a change in the alternative splice isoform distribution of FasII. We demonstrate that only transmembrane isoforms of FasII are required for normal synapse development and show that restoration of one specific transmembrane isoform can rescue beag mutant synaptic morphological defects. Our data reveal that Beag and Dsmul are essential alternative splicing factors required for normal synaptic development and the regulation of FasII.

\section{Materials and Methods}

Drosophila stocks

The following Gal4 lines were used: OK6-Gal4, G14-Gal4 (Aberle et al., 2002), C155-Gal4 (Lin and Goodman, 1994), OK371-Gal4 (Mahr and Aberle, 2006), OK319-Gal4 (B.J. Choi and B.D. McCabe, unpublished observations), D42-Gal4 (Brand and Perrimon, 1993), and Da-Gal4 (Wodarz et al., 1995). We use the following nomenclature: FasII-APEST+ (Lin and Goodman, 1994; FasII-PA Flybase), FasII-TM-APEST - (Lin and Goodman, 1994), FasII-C (FasII-PC Flybase; Grenningloh et al., 1991), and FasII-B (FasII-RB Flybase). The following FasII lines were used: UAS-FasII-A-PEST + (Schuster et al., 1996a), UASFasII-A-PEST- (Lin and Goodman, 1994), UAS-FasII-C (Lin and Goodman, 1994; D. Lin and C.S. Goodman, unpublished observations), UAS-FasII-A-PEST + AAE (Zito et al., 1997; K. Zito and C.S. Goodman, unpublished observations), UAS-CD8-FasII-A-PEST+Intra (Zito et al., 1997), fasII ${ }^{e 76}$, fasII ${ }^{e B 112}$ (Grenningloh et al., 1991), Df(1)BSC869 (K. Cook, S, Christensen, personal communication to Flybase), UASFasIIRNAi-total (Dietzl et al., 2007; Vienna Drosophila RNAi Centre, \#v103807),UAS-FasIIRNAi-A, UAS-RNAiResistantFasII-A-PEST+, and UAS-RNAiResistantFasII-A-PEST - (construction described below). To confirm the sequence of the UAS-FasII-C transgene, genomic DNA was isolated from the transgenic flies and primers specific to the pUAST vector (UAST-FWD GAGCGCCGGAGTATAAATAGAGG and UASTREV CTCCCATTCATCAGTTCCATAGGT) were used to PCR-amplify the transgene. The sequence of this PCR product corresponded to the entire coding sequence of FasII-C as in Flybase, with the exception of several single base pair substitutions that do not alter the amino acid sequence. The following beag lines were used: $\mathrm{Df}(3 \mathrm{R})$ Exel6151 (Thibault et al., 2004), $P[E P] C G 18005^{E P 3260}$ (Rørth, 1996), beag ${ }^{I}$, UAS-EYFPBeag, UAS-Beag, and Genomic beag (construction described below). The following $d s m u 1$ lines were used: $\operatorname{Df}(3 \mathrm{R})$ Exel6182 (Thibault et al., 2004), Pbac [WH]CG5451 f03090 (Thibault et al., 2004), UAS-myc-tagRFP2Dsmu1, and UAS-Dsmu1 (construction described below). Animals of either sex were analyzed for mutants or constructs on autosomes. For hemizygous or heterozygous mutants on the $\mathrm{X}$ chromosome, males or females were selected as appropriate. The number of animals analyzed per genotype is detailed in Table 1.

\section{Construction of transgenes}

UAS-EYFPBeag, UAS-Beag. The Beag open reading frame (ORF) was amplified from the LD21347 cDNA clone (Stapleton et al., 2002; Berkeley Drosophila Genome Project, BDGP) by PCR and subcloned into a pUAST vector with or without a sequence encoding EYFP (B,D.M., unpublished observations). Transgenic flies with insertions on chromosome II were generated by standard P-element techniques.

Genomic beag. The beag genomic rescue construct was generated by PCR amplification of the beag gene from genomic DNA using a forward primer $1 \mathrm{~kb}$ upstream of the transcription start site and a reverse primer $0.5 \mathrm{~kb}$ downstream of the 3'UTR (primers beag-GR-F CACCAGACCGAAAGTTTCCGCAGCA and beag-GR-R TTTGGATCCCCCGCGAAGGTAATTACATTT). A $3 \times$ Flag tag was inserted immediately upstream of the beag start codon, and a stop codon was inserted at the 40th base pair of the ORF of the upstream gene ada to avoid ada overexpression artifacts. This sequence was subcloned into the pBID vector (J. Wang, E.S. Beck, and B.D. McCabe, unpublished observations). Transgenic flies with insertions on chromosome II at the attP51D landing site were generated using Phi3C1 transgenesis (Groth et al., 2004).

UAS-myc-tagRFP-Dsmu1, UAS-Dsmu1. The Dsmul ORF was amplified from the LD41216 cDNA clone ((Rubin et al., 2000; BDGP) by PCR (primers Smu 1-F CACCATGTCCATAGAAATCGAATCA and Smul-R AAACTCGAGCCACCAACTAAAACTAACTAG), TOPO cloned into pENTR, and then Gateway cloned into pBID-UASC-GW and pBIDUASC-MRG-GW (J. Wang, E.S. Beck, and B.D. McCabe, unpublished observations). Transgenic flies with insertions on chromosome II at the attP40 landing site were generated using Phi3C1 transgenesis (Groth et al., 2004).

UAS-FasIIRNAi-A. UAS-FasIIRNAi-A was generated by PCR amplification of the sequence corresponding to FasII exon 12 from cDNA made from larval brains (primers FasII-RNAi-A-F, CACCCGTCATCC AAGTGGCTGAGCG and FasII-RNAi-A-R, GCTTGGCCTCGTCGTC GATTT). The PCR product was TOPO cloned into pENTR and then Gateway cloned into pBID-UAS-GGi (J. Wang, E.S. Beck, and B.D. McCabe, unpublished observations) such that each product was inserted twice, in opposite directions, immediately downstream of a UAS sequence and with a $\mathrm{ftz}$ intron sequence in between the two inserts, allowing the formation of a hairpin RNA when transcribed. Transgenic flies with insertions in the attP2 on chromosome III site were generated using Phi3C1 transgenesis (Groth et al., 2004).

UAS-RNAiResistantFasII-A-PEST+, UAS-RNAiResistantFasII-APEST - . A modified version of the final $101 \mathrm{bp}$ of the FasII-A-PEST + and A-PEST - ORF were synthesized and inserted in the pUC57 vector (GenScript) such that the last base of every codon was altered to maintain the amino acid sequence while inhibiting targeting by FasIIRNAi-A.

The sequence of this synthesized fragment was as follows: GGTACCCC CTTAAGGACGCCTACGGGAAGTATAAAGCAAAATTCCACGATAGA GTTTGATGGCCGTTTTGTACATTCCCGGAGCGGAGAAATTATAGG CAAAAACTCTGCAGTCTAATCTAGA.

The rest of the FasII-A-PEST + and FasII-A-PEST - coding sequences were amplified individually from cDNA made from larval brains (primers FasIIRNAiRes-F CACCGAATTCAACATGGGTGAATTGCCGCCAAAT, FasIIRNAiRes-F-PEST + R AAGCTTCTAGAGCGTCCTTAAGGGCTCC TTTTCGTCCAATGGCGTGC, and FasIIRNAiRes-F-PEST-R AAGCTT CTAGAGCGTCCTTAAGGGCTCCTTTTCGTCCCTGCCCAGC) and cloned into the pCMV-tag5a vector using EcoRI and HindIII restriction sites included in the primers. The mutated $3^{\prime}$ sequence was subcloned into a plasmid containing the rest of the coding sequence using engineered AflII and $\mathrm{XbaI}$ restriction sites, and then the entire FasII coding sequence was subcloned into the pBID-UASC vector using introduced EcoRI and HindIII restriction sites (J. Wang, E.S. Beck, and B.D. McCabe, unpublished observations). Transgenic flies with insertions on chromosome II at the attp40 landing site were generated using Phi3C1 transgenesis (Groth et al., 2004).

\section{Immunohistochemistry}

Wandering third instar larvae were collected, dissected, and stained as previously described (Brent et al., 2009a,b). Primary antibodies used were mouse anti-Bruchpilot (Wagh et al., 2006)(1:500, NC82; Developmental Studies Hybridoma Bank, DSHB), mouse anti-cysteine string protein (CSP; Zinsmaier et al., 1990) (1:200; DSHB ), rabbit anti-DAP160 (1:100; Marie et al., 2004), mouse anti-DLG (Parnas et al., 2001)(1:200; from C.S. Goodman), rat anti-elav (1:500; DSHB), mouse anti-FasII TM (mAb 1D4, 1:900; DSHB, from C.S. Goodman), mouse anti-FasII total (mAb 34B3, 1:20; DSHB), mouse anti-Flag (1:500; Sigma), chicken antiGFP (1:1000; Abcam), mouse anti-Map1B (1:100; Hummel et al., 2000), mouse anti-myc (1:400; DSHB), mouse anti-neuroglian (Hortsch et al., 1990)(1:100; DSHB), guinea pig anti-phospho-Mad (1:1000; gift from $E$. Laufer), mouse anti-synaptotagmin (1:1000, mAb 3H2 2D7; DSHB), and Cy5-conjugated goat anti-horseradish peroxidase (HRP) (1:400; Jackson ImmunoResearch). Conjugated secondary antibodies were used (1:1000, Jackson ImmunoLabs; 1:2000, Invitrogen). Larval preparations were imaged on a Zeiss LSM 510 Confocal microscope. Relative immunohistochemistry intensity measurements were made using these images. The average intensity of staining of the antibody of interest was calculated within the synaptic area colabeled by anti-HRP, which labels neuronal membranes. Synaptic boutons at muscle 4 of segment A3 were used for intensity measurements and calculations were done using MetaMorph software (Molecular Devices). 
Table 1. Morphological data

\begin{tabular}{|c|c|c|c|c|c|c|c|}
\hline Genotype & Control & N & bouton number & MSA $\left(10^{4} \times \mu \mathrm{m}^{2}\right)$ & Bouton number/MSA & Bouton number/MSA (norm) & Pv control (ANOVA) \\
\hline Canton S (CS) & - & 71 & $50.35 \pm 1.37$ & $18.65 \pm 1.84$ & $3.01 \pm 0.09$ & $1.00 \pm 0.03$ & - \\
\hline 7A6 (MHC-CD8Sh-GFP) & - & 69 & $41.80 \pm 1.73$ & $15.85 \pm 0.48$ & $3.05 \pm 0.15$ & $1.00 \pm 0.05$ & - \\
\hline $\mathrm{beag}^{7} / \mathrm{beag}^{7}$ & $7 A 6$ & 67 & $28.31 \pm 1.32$ & $14.01 \pm 0.36$ & $2.10 \pm 0.09$ & $0.69 \pm 0.03$ & $<0.001$ \\
\hline beag $^{1} /$ Df6151 & 7A6/w1118 & 43 & $29.88 \pm 1.66$ & $16.33 \pm 0.40$ & $1.87 \pm 0.13$ & $0.60 \pm 0.04$ & $<0.001$ \\
\hline $\mathrm{beag}^{1} /$ Df6151 female & 7A6/w1118 & 40 & $26.58 \pm 0.53$ & $18.34 \pm 0.51$ & $1.34 \pm 0.10$ & $0.50 \pm 0.02$ & $<0.001$ \\
\hline 7A6/CS & - & 43 & $41.49 \pm 1.67$ & $14.43 \pm 0.47$ & $2.94 \pm 0.12$ & $1.00 \pm 0.04$ & - \\
\hline dsmu $7^{\text {pBacto3090 } / D f 6182 ~}$ & CS & 39 & $31.05 \pm 1.27$ & $14.92 \pm 0.39$ & $2.13 \pm 0.10$ & $0.71 \pm 0.03$ & $<0.001$ \\
\hline beag $^{E P 3260} / \mathrm{Df6} 151$ & CS & 45 & $30.84 \pm 1.19$ & $13.78 \pm 0.38$ & $2.31 \pm 0.11$ & $0.77 \pm 0.04$ & $<0.001$ \\
\hline Beag $^{E P 3260}$, Df6182/dsmu $7^{\text {pBacf030990 }}$,Df6151 & CS & 48 & $24.13 \pm 1.16$ & $11.57 \pm 0.39$ & $2.16 \pm 0.11$ & $0.72 \pm 0.04$ & $<0.001$ \\
\hline 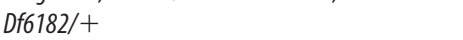 & CS & 47 & $54.15 \pm 2.31$ & $20.81 \pm 0.59$ & $2.64 \pm 0.10$ & $0.88 \pm 0.03$ & $<0.05$ \\
\hline $\mathrm{beag}^{1} /+$ & CS & 44 & $69.14 \pm 1.87$ & $25.15 \pm 0.60$ & $2.80 \pm 0.09$ & $0.90 \pm 0.03$ & ns \\
\hline $\mathrm{beag}^{7} / \mathrm{Df6} 6182$ & CS & 36 & $55.92 \pm 2.43$ & $24.49 \pm 0.56$ & $2.31 \pm 0.10$ & $0.77 \pm 0.03$ & $<0.001$ \\
\hline OK6G4/UASDsmu1; dsmu1 $1^{\text {pBacf03090 } / D f 6182 ~}$ & CS & 40 & $40.33 \pm 1.61$ & $13.80 \pm 0.59$ & $3.10 \pm 0.17$ & $1.02 \pm 0.06$ & ns \\
\hline C155G4; UASBeag; dsmu1 $7^{\text {pBacf03090 } / D f 6182}$ & CS & 62 & $44.44 \pm 1.39$ & $17.28 \pm 0.37$ & $2.64 \pm 0.10$ & $0.87 \pm 0.03$ & $<0.05$ \\
\hline G14G4/UASBeag; beag /Df6151 & 7A6/w1118 & 47 & $41.57 \pm 1.99$ & $17.73 \pm 0.47$ & $2.35 \pm 0.10$ & $0.76 \pm 0.03$ & $<0.001$ \\
\hline G14G4/UASDsmu1; dsmup pBacf03090/Df6182 & CS & 44 & $32.07 \pm 1.73$ & $21.47 \pm 0.36$ & $1.51 \pm 0.08$ & $0.49 \pm 0.03$ & $<0.001$ \\
\hline CS female & - & 47 & $54.70 \pm 2.33$ & $19.40 \pm 0.47$ & $2.85 \pm 0.12$ & $1.00 \pm 0.04$ & - \\
\hline fas $/ l^{e 76} /+$ & CS female & 85 & $46.61 \pm 1.72$ & $17.27 \pm 0.39$ & $2.67 \pm 0.07$ & $0.94 \pm 0.02$ & ns \\
\hline fasll/ ${ }^{e B 112} /+$ & CS female & 46 & $55.15 \pm 1.62$ & $20.28 \pm 0.58$ & $2.78 \pm 0.09$ & $0.97 \pm 0.03$ & ns \\
\hline DfBSC869/+ & CS female & 47 & $61.17 \pm 2.27$ & $20.63 \pm 0.66$ & $3.04 \pm 0.12$ & $1.07 \pm 0.04$ & ns \\
\hline fas $11^{276 / e 76}$ & CS female & 47 & $29.72 \pm 1.24$ & $20.78 \pm 0.39$ & $1.43 \pm 0.05$ & $0.50 \pm 0.02$ & $<0.001$ \\
\hline fas $^{e 76 / e B 112}$ & CS female & 48 & $33.73 \pm 0.91$ & $23.72 \pm 0.54$ & $1.44 \pm 0.04$ & $0.50 \pm 0.01$ & $<0.001$ \\
\hline fas// ${ }^{e 76} / D f B S C 869$ & CS female & 47 & $39.10 \pm 1.55$ & $23.31 \pm 0.32$ & $1.70 \pm 0.07$ & $0.60 \pm 0.03$ & $<0.001$ \\
\hline 7A6/CS female & - & 43 & $50.51 \pm 1.80$ & $17.75 \pm 0.80$ & $3.01 \pm 0.13$ & $1.00 \pm 0.04$ & - \\
\hline UASRNAiResFasIITMB/UASdcr2; FasIIRNAiTM/daG4 & UASdcr2/+; daG4/+ & 47 & $65.72 \pm 2.31$ & $23.98 \pm 0.75$ & $2.84 \pm 0.12$ & $1.08 \pm 0.05$ & ns \\
\hline fas $^{e 76} /$ DfBSC869; OK6G4/UASFasIIGPI & CS female & 46 & $38.87 \pm 1.58$ & $22.64 \pm 0.65$ & $1.77 \pm 0.08$ & $0.62 \pm 0.03$ & $<0.001$ \\
\hline 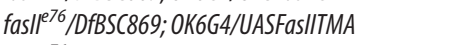 & CS female & 43 & $71.65 \pm 2.83$ & $25.56 \pm 0.60$ & $2.86 \pm 0.13$ & $1.00 \pm 0.04$ & ns \\
\hline fasII" $^{e 76} / D f B S C 869 ; 0 K 6 G 4 / U A S F a s I I T M B$ & CS female & 47 & $59.5 \pm 2.44$ & $23.33 \pm 0.67$ & $2.63 \pm 0.13$ & $0.92 \pm 0.04$ & ns \\
\hline OK6G4/UASFasII-GPI & 7A6/w1118 & 42 & $57.93 \pm 2.65$ & $20.50 \pm 0.43$ & $2.88 \pm 0.15$ & $0.98 \pm 0.05$ & ns \\
\hline OK6G4/UASFasII-GPI; beag ${ }^{1} /$ ff6151 & 7A6/w1118 & 44 & $30.43 \pm 1.58$ & $15.45 \pm 0.51$ & $2.08 \pm 0.14$ & $0.71 \pm 0.05$ & $<0.001$ \\
\hline OK6G4/UASFasII-TMB & 7A6/w1118 & 38 & $80.92 \pm 3.10$ & $20.29 \pm 0.47$ & $4.08 \pm 0.19$ & $1.39 \pm 0.07$ & $<0.001$ \\
\hline OK6G4/UASFasII-TMB; beag ${ }^{1} / D f 6151$ & 7A6/w1118 & 46 & $32.96 \pm 1.33$ & $15.32 \pm 0.37$ & $2.19 \pm 0.09$ & $0.74 \pm 0.03$ & $<0.01$ \\
\hline OK6G4/UASFasII-TMA & 7A6/w1118 & 42 & $51.21 \pm 3.33$ & $15.76 \pm 0.64$ & $3.30 \pm 0.18$ & $1.09 \pm 0.06$ & ns \\
\hline OK6G4/UASFasII-TMA; beag ${ }^{7}$ Df6151 & 7A6/w1118 & 39 & $44.05 \pm 2.01$ & $13.50 \pm 0.48$ & $3.37 \pm 0.18$ & $1.14 \pm 0.06$ & ns \\
\hline OK6G4/UAS-CD8-FasIITMAIntra & 7A6/w1118 & 78 & $56.78 \pm 1.66$ & $19.06 \pm 0.46$ & $2.88 \pm 0.11$ & $1.03 \pm 0.02$ & ns \\
\hline OK6G4/UAS-CD8-FasIITMAIntra; beag /Df6151 & 7A6/w1118 & 104 & $43.94 \pm 1.26$ & $15.31 \pm 0.26$ & $2.91 \pm 0.08$ & $0.94 \pm 0.05$ & ns \\
\hline C155G4/UASFasII-TMA;dsmu1 $1^{\text {pBaco3090 } / D f 6182 ~}$ & CS & 45 & $43.64 \pm 1.91$ & $17.68 \pm 0.49$ & $2.52 \pm 0.12$ & $0.83 \pm 0.04$ & $<0.001$ \\
\hline
\end{tabular}

Morphological analysis of $\mathrm{NMJS}$

Wandering third instar larvae were stained with antibodies against CSP and HRP (described above). All morphological analysis was done at muscle 4 of segment A3. Type Ib and Is boutons were counted using a $40 \times$ objective on a Zeiss Axio Imager.Z1 microscope. Muscle area was measured using a $20 \times$ objective micrometer. Raw bouton and muscle surface area for all genotypes analyzed are in Table 1. Synaptic area measurements were made with MetaMorph software on images obtained by confocal microscopy. Total presynaptic area was determined by calculating the area of all type Ib boutons on a single muscle 4 , measured by the area stained by CSP. Total area was divided by bouton number to determine average bouton area. At least 35 synapses were analyzed per genotype.
Time-lapse live imaging

NMJs were visualized using MHC::hCD8-GFP-Shaker protein in control and beag mutant larvae (Zito et al., 1999). Animals were anesthetized by $\sim 15$ min exposure to a vapor mixture of 35\% methyl salicylate and $16 \%$ menthol (Haw Par Healthcare) at the second instar and again $48 \mathrm{~h}$ later at the third instar. For imaging, larvae were placed on a slide with $70 \%$ glycerol and a coverslip. Imaging time was limited to $<30 \mathrm{~min}$ after which animals were washed gently with PBS, allowed to recover, and returned to the food media. Mutants and corresponding controls were imaged on the same day in a random order to minimize handling variability. Only images from animals that survived the entire $2 \mathrm{~d}$ imaging procedure were included in analysis. Images were collected using a Zeiss Z1 Apotome system using a $63 \times$ lens. To count bouton addition in live 
images only new distal boutons, which can be reliably recognized by live imaging, were used for analysis.

\section{Active zone quantification}

To determine the number of active zones per NMJ, wild-type and $\mathrm{beag}^{l} / \mathrm{Df}$ larvae were dissected and stained as described above with antiBruchpilot. The number of discrete anti-Bruchpilot-stained active zones per NMJ were counted using an E600 Nikon epifluorescence microscope with a Plan Apo $\times 100 / 1.4$ NA objective. Counting was done for the NMJ synaptic terminal on muscle 4 of segment A3.

\section{Electrophysiology}

Intracellular recording from muscle 6 , segment A3 was performed as previously described (Imlach and McCabe, 2009). Third instar larvae were dissected in HL3 (Stewart et al., 1994) and recordings were performed in HL3 containing $1 \mathrm{~mm} \mathrm{Ca}^{2+}$ (except where noted). Data were only analyzed when the resting membrane potential was $<-55 \mathrm{mV}$. Evoked junctional potential (EJP) amplitude was analyzed using Clampex v 8.2.0.235 software (Molecular Devices). Miniature excitatory junctional potential (mEJP) amplitude and frequency were analyzed using Mini analysis software (Synaptosoft, v 6.0.3). Recordings were done from a minimum of 10 muscles per genotype.

\section{In situ hybridization}

In situ hybridization of Drosophila embryos was performed as previously described (Kosman et al., 2004), with the following modifications. After incubation in anti-Dig-AP Fab fragments antibody (1:500; Roche), in situs were developed for 10-30 min using the BCIP/NBT Alkaline Phosphatase Substrate Kit IV (Vector Laboratories). Antisense and sense beag RNA probes were transcribed in vitro from PCR products amplified from the LD21347 cDNA clone (Stapleton et al., 2002; BDGP). The entire beag ORF was amplified using the $5^{\prime}$ primer TAATACGACTCACTATAGGG AGAACGCGCTACAATTAACATAAC and the $3^{\prime}$ primer GCAGATCT GATATCATCGCCACT for the antisense probe and the $5^{\prime}$ primer ACGC GGCTACAATTAATACATAACC and the $3^{\prime}$ primer AGCCGATTCATT AATGCAGGT for the sense (negative control) probe.

\section{Quantitative real-time PCR}

RNA was isolated from third instar larval brains using TRIzol and isopropanol precipitation. RNA samples were DNase treated (TURBO DNA-free Kit; Applied Biosystems) and reverse transcribed (SuperScript III First-Strand Synthesis System for RT-PCR; Invitrogen). Quantitative PCR was performed using an Eppendorf Mastercycler ep realplex Thermal Cycler.

The Primer 3 web site (http://fokker.wi.mit.edu/primer3/input.htm) was used to design primers for qPCR that spanned exon-exon junctions to avoid amplification of genomic DNA. The following primers pairs were used:

RP49: F-CATCCGCCCAGCATACAG, R-CCATTTGTGCGACAGC TTAG.

FasII-A: F-TACTGTCCGGGCGTTAAGAT, R-ACGTCAATTCCTCG TGTCGT, FasII-C: F-TACTGTCCGGGCGTTAAGAT, R-GAATCGGACT CACCTCGTGT, FasII total: F-CAACCAGGTGGGATTAGGAA, R-TAAC GCCCGGACAGTATTTG.

qRT-PCRs was performed at least three times for each of at least three biological replicates per genotype. ddCt was calculated for each FasII primer set, using rp49 as a housekeeping gene for normalization.

\section{Semiquantitative PCR}

cDNA isolated from larval brains of wild-type and beag mutant larvae was amplified with a $5^{\prime}$ primer in the last common FasII exon and a $3^{\prime}$ primer in the shared 3' UTR of FasII-A-PEST + and FasII-A-PEST-. This PCR yielded a slower migrating band corresponding to FasII-A$\mathrm{PEST}+$ and a faster migrating band corresponding to FasII-A-PEST - by agarose gel electrophoresis. Gels were photographed and the intensity of the FasII-A-PEST + and PEST - bands and the background intensity were measured from the same image using ImageJ. The background intensity was subtracted from the band intensities and the ratio of the intensity of the two bands was calculated. Four biological replicates of wild-type and beag cDNA were used and for each set the PCR was done one to three times, leading to a final $N$ of 7 .

\section{Western blotting}

Samples for Western blots were prepared by putting 5-10 whole larvae, dissected larval brains, or dissected larval body walls directly into sample buffer.

The primary antibodies used were as follows: mouse anti-elav (1:500, mAb 9F8A9; DSHB), mouse anti-FasII TM (mAb 1D4, 1:900; DSHB, from C.S. Goodman), and mouse anti-FasII total (mAb 34B3, 1:20; DSHB). Peroxidase-conjugated goat anti-rabbit (1:5000; Jackson ImmunoLabs) was used as a secondary antibody.

\section{Statistical analysis}

For all comparisons, statistical significance was calculated using ANOVA.

\section{Results}

Mutations in the gene beag lead to a decrease in NMJ synaptic bouton number and increased bouton size

During larval development, Drosophila NMJs grow by the iterative addition of synaptic boutons (Zito et al., 1999; Prokop, 2006). On average these synaptic boutons have a diameter of $\sim 3$ $\mu \mathrm{m}$ and bouton diameters rarely exceed $6 \mu \mathrm{m}$ (Johansen et al., 1989). When normalized to muscle surface area, the number of synaptic boutons at each NMJ terminal is also highly stereotyped (Schuster et al., 1996a). From a forward genetic screen for mutants with aberrant synapse development (McCabe et al., 2004), we identified a novel mutant beag (pronounced "be-yug," Gaelic for small or petite). beag mutants were adult semilethal with $39 \%$ of the expected number of animals surviving to adulthood. beag mutant larvae had decreased numbers of NMJ synaptic boutons compared with wild type, but a significant increase in the area of the boutons present (Fig. 1 $A, B$ ). To characterize beag mutant NMJs, we used labeling with an antibody against the synaptic vesicle protein CSP (Zinsmaier et al., 1994) to count the number of synaptic boutons as well as measure the total presynaptic bouton area. We divided the total presynaptic bouton area by the number of synaptic boutons to measure the average bouton area. beag mutants had a 39\% decrease $(p<0.001)$ in the number of synaptic boutons while the average bouton area was dramatically increased to $180 \%$ of wild type ( $p<0.001)$ (Fig. $1 C, D$, Table 1$)$. Thus the total presynaptic area was increased by $21 \%(p<0.01)$ compared with wild type (Fig. $1 E$ ). This result suggested to us that the process of bouton addition might be defective beag mutants. To directly examine this process, we imaged NMJ terminals by time-lapse live imaging through the transparent cuticle of whole animals in wild type and beag strains that expressed CD8-GFP-Sh, a marker of postsynaptic NMJ membranes (Zito et al., 1999). We anesthetized animals at the second larval instar, imaged their NMJ terminals, returned them to the food media, and then imaged the terminal again $48 \mathrm{~h}$ later at the third larval instar. To measure new bouton formation, we restricted our analysis to the addition of distal boutons, which can be reliably detected by live imaging. After $48 \mathrm{~h}$, $51.4 \%$ of wild-type NMJ terminals had new boutons added to the distal end of NMJ terminals (Fig. $1 F$ ). In contrast, only $6.7 \%(p<0.001)$ of beag mutant NMJs had new distal boutons added after $48 \mathrm{~h}$ (Fig. 1G). These results indicated that beag mutants had a failure of new bouton addition coupled with aberrant morphological expansion of existing boutons.

The beag point mutation $\left(\right.$ beag $\left.^{l}\right)$ was mapped by complementation to the deficiency $\operatorname{Df}(3 \mathrm{R})$ Exel6151, covering the cytological region 85C3-85C11 (Thibault et al., 2004). Sequencing of genes covered by this deficiency led to the identification of a point 

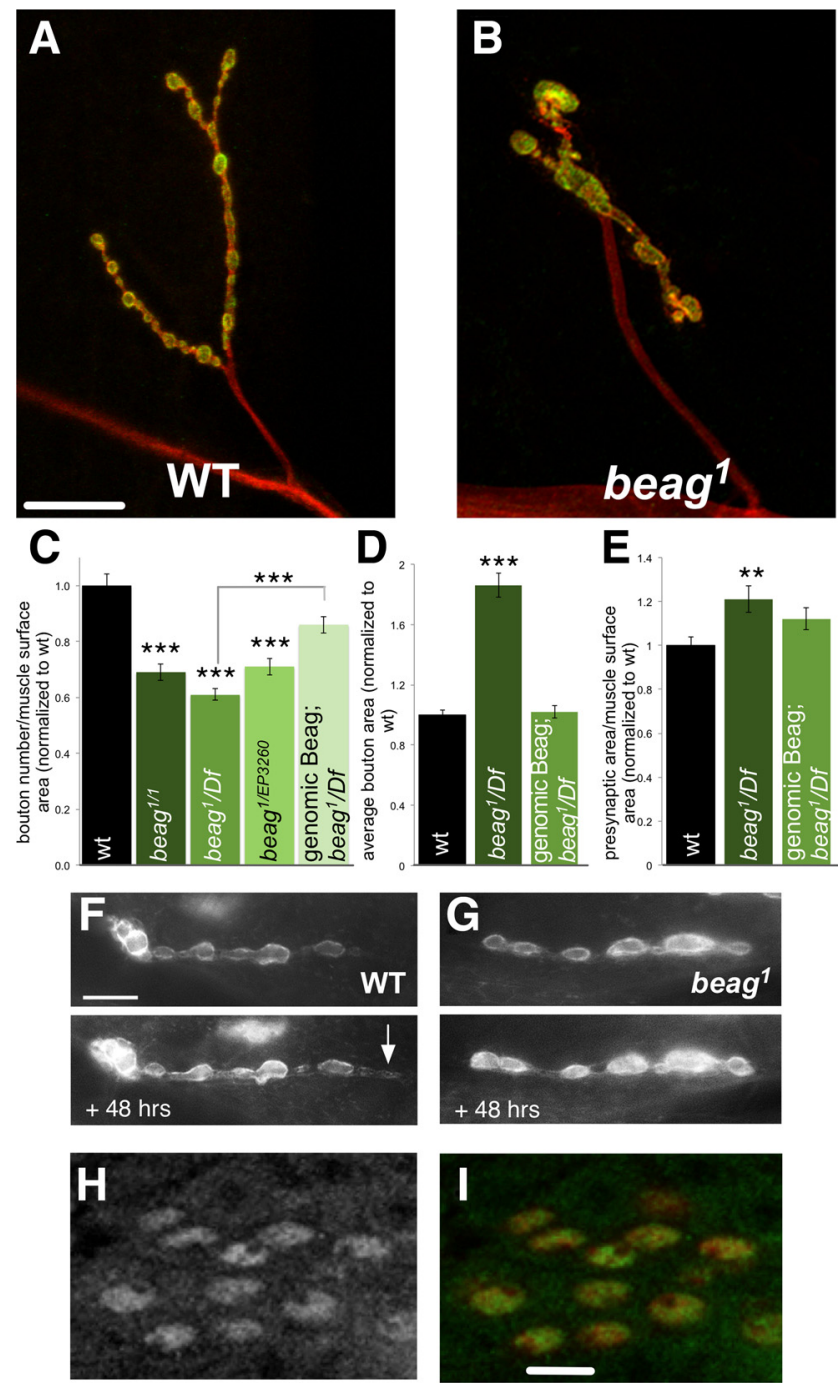

Figure 1. beag mutants have decreased NMJ synaptic bouton number and increased synaptic bouton area. $\boldsymbol{A}, \boldsymbol{B}$, Representative images of NMJ synaptic terminals at muscle 4 of segment $A 3$ of third instar wild-type and beag ${ }^{1 / 1}$ mutant larvae stained with anti-CSP (green) to label the presynapse and anti-HRP (red) to label the neuronal membrane. Scale bar, $20 \mu \mathrm{m}$. beag mutants have a reduction in synaptic bouton number $(\boldsymbol{C})$, an increase in average synaptic bouton area $(\boldsymbol{D})$, and an increase in total presynaptic area $(\boldsymbol{E})$. Expression of a genomic Beag transgene rescues beag mutant bouton number $(\boldsymbol{C})$, beag mutant bouton area $(\boldsymbol{D})$, and total presynaptic area $(\boldsymbol{E})$. C, Quantification of bouton number normalized to muscle surface area. $\boldsymbol{D}$, Quantification of average bouton area. $\boldsymbol{E}$, Quantification of total presynaptic terminal area normalized to muscle surface area. Live imaging of developing NMJ synaptic terminals labeled with CD8-GFP-SH in wild-type $(\boldsymbol{F})$ and beag ${ }^{7}$ mutant $(\boldsymbol{G})$ larvae at the second larval instar and after $48 \mathrm{~h}$ at the third larval instar. A new distal bouton addition is indicated by the arrow. Scale bar, $8 \mu \mathrm{m}$. $\boldsymbol{H}, \boldsymbol{I}$, Flag-tagged genomic Beag localizes to the nucleus $(\boldsymbol{H})$ and colocalizes with Elav in neurons ( $\boldsymbol{I}$, Beag green, Elav red). Scale bar, $10 \mu \mathrm{m}$. Error bars indicate SEM. ${ }^{* *} p<0.01,{ }^{* * *} p<0.001$, significance calculated versus wild-type control except where indicated.

mutation in the previously uncharacterized gene CG18005, which we renamed beag. This mutation introduces a premature stop codon at amino acid 304 of the predicted 557 aa protein. We also identified a P element, EP3260, inserted at the 94th nucleotide of the beag ORF as an additional beag allele (Rørth, 1996). beag $^{1} / \mathrm{Df}(3 \mathrm{R})$ Exel6151 and beag $^{1} /$ beag $^{E P 3260}$ mutants recapitulated the synaptic structural phenotypes of beag ${ }^{1}$ homozygotes (Fig. 1C), suggesting that the beag ${ }^{l}$ mutation is a strong loss-offunction allele of CG18005. For all subsequent experiments, ex- cept where noted, we used the beag $^{1} / \mathrm{Df}(3 \mathrm{R})$ Exel6151 mutant combination.

Beag is a broadly expressed nuclear protein

In situ hybridization of embryos with RNA probes against the beag transcript showed broad expression of beag message, including expression in the CNS (data not shown). We generated an epitope-tagged beag transgene "genomic Beag" that includes the entire beag gene, with $1 \mathrm{~kb}$ of DNA upstream of the transcription start site and $0.5 \mathrm{~kb}$ of DNA downstream of the beag 3'UTR. This construct, which rescued beag mutant synapse morphology defects and beag mutant viability (Fig. $1 C-E$, data not shown), revealed that Beag protein is expressed in many tissues in the larva. Beag protein was localized to the nuclei of neurons, where it colocalized with the neural nuclear protein Elav (Fig. $1 H, I$ ). The nuclear localization of Beag was confirmed at higher resolution with an EYFP-tagged, Gal4-driven beag cDNA transgene (data not shown). Genomic Beag expression was also observed in the nuclei of non-neural cells, including muscles (data not shown).

\section{Beag encodes a spliceosomal protein}

Sequence analysis of the Beag protein revealed that it was most similar to RED protein in humans (Assier et al., 1999) and SMU-2 protein in C. elegans (Spartz et al., 2004). RED is a ubiquitously expressed nuclear protein that was purified from human spliceosomes. Recently, Beag was also found to be a constituent of Drosophila spliceosomes (Herold et al., 2009). The C. elegans homolog of Beag, SMU-2, which is also expressed in the nuclei of all cells (Spartz et al., 2004), was first identified as a regulator of unc-52 alternative splicing. smu-2 loss-of-function mutants can suppress lethal point mutations in $u n c-52$ by altering the alternative splicing of an UNC-52 exon. Alone, mutants of smu-2 have mild phenotypes including decreased mobility, growth, and brood size; however, no neuronal phenotype has been reported (Lundquist and Herman, 1994; Spartz et al., 2004). Like RED and SMU-2, Beag does not have a recognizable RNA binding motif. These proteins may influence alternative splicing through interaction with other RNA binding proteins (Black, 2003). The presence of Beag in the Drosophila spliceosome and its homology to SMU-2, which has a demonstrated role in alternative splicing, suggested that Beag might regulate synaptic development through the regulation of pre-mRNA splicing.

\section{Dsmul and Beag have similar synaptic phenotypes and function in the same genetic pathway}

In the same study in which C. elegans smu-2 was identified, a second splicing regulator, $s m u-1$, was also isolated. $s m u-1$ and smu-2 mutants have similar phenotypes, both alter $u n c-52$ alternative splicing, and SMU-1 and SMU-2 proteins physically interact (Lundquist and Herman, 1994; Spartz et al., 2004). Like SMU-2, SMU-1 protein is located in the nucleus and is ubiquitously expressed (Spike et al., 2001). The human protein most similar to SMU-1 is known as fSAP-57 or SMU1 and is enriched in the brain (Di Benedetto et al., 2001; Spike et al., 2001). This protein was purified from human spliceosomes (Zhou et al., 2002) and has also been shown to regulate alternative splicing (Sugaya et al., 2006). We hypothesized that Beag might regulate synaptic development as part of a conserved complex with SMU1, and we sought to identify the Drosophila homolog of this protein. We examined the Drosophila genome and determined that the protein most similar to C. elegans SMU-1 and human SMU1 was encoded by the previously uncharacterized gene CG5451. Similar to Beag, the product of this gene is also a com- 

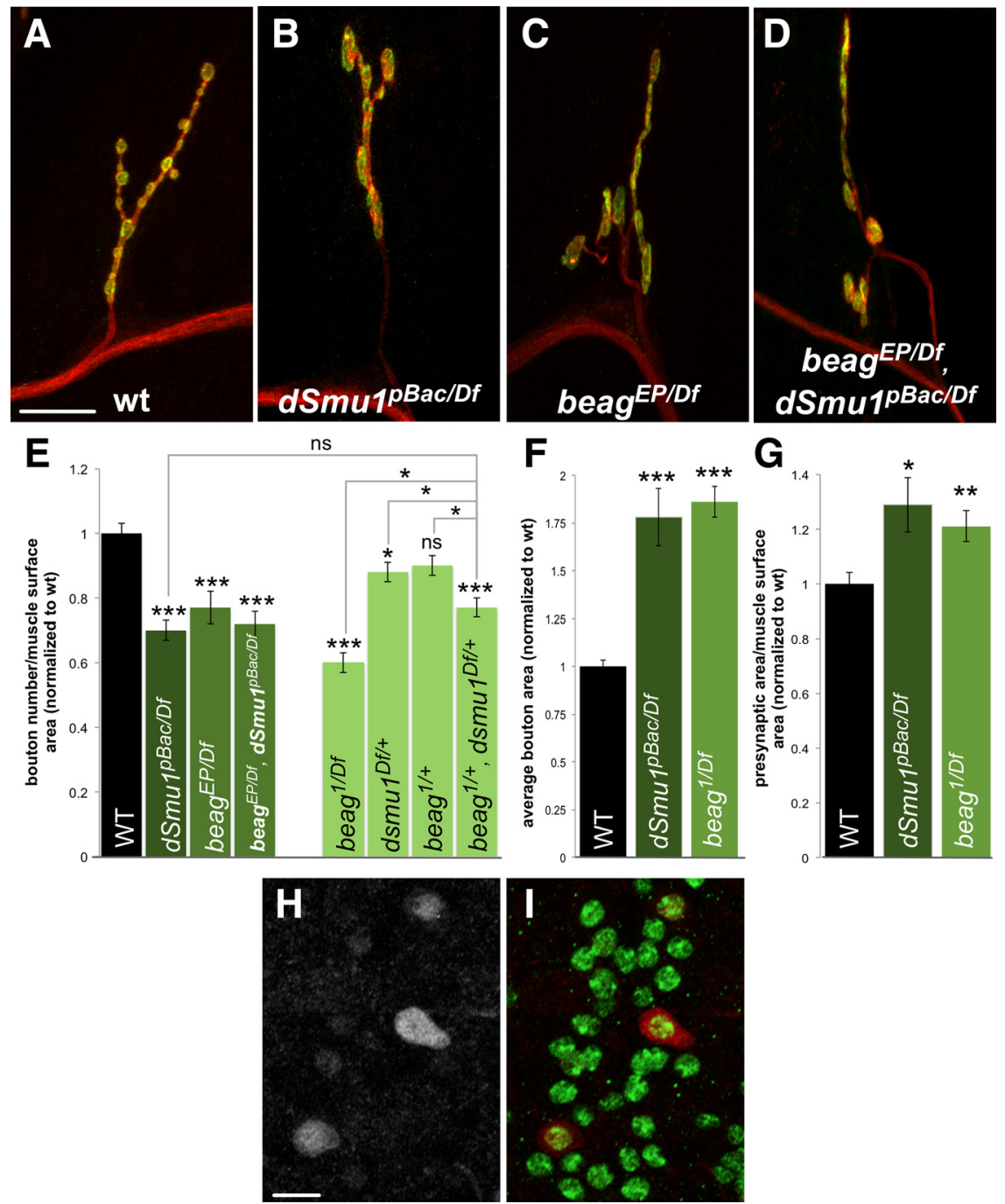

Figure 2. beag and dsmu-1 mutants have similar NMJ synaptic morphology phenotypes. dsmu $1^{\text {pBacf03090 }}$ Df mutants (B) have decreased synaptic bouton number, no change in total presynaptic area, and increased synaptic bouton area compared with wild type $(\boldsymbol{A})$. These phenotypes are similar to those seen in beag ${ }^{E P 3260} / \mathrm{Df}$ mutants $(\boldsymbol{C})$. beag ${ }^{E P / D f} \mathrm{dsmu}^{\text {PBac/Df }}$ double mutants have similar phenotypes to beag and dsmu 1 single mutants $(\boldsymbol{D})$. beag $^{1 /+}, d s m u 1^{D f /+}$ trans-heterozygotes have a similar decrease in bouton number as either heterozygote alone $(\boldsymbol{E})$. $\boldsymbol{A}-\boldsymbol{D}$, Representative images are NMJ synaptic terminals at muscle 4 of segment A3 stained with anti-CSP (green) to label the presynapse and anti-HRP (red) to label the neuronal membrane. Scale bar: (in $\boldsymbol{A}) \boldsymbol{A}-\boldsymbol{D}$, $20 \mu \mathrm{m}$. $\boldsymbol{E}$, Quantification of bouton number normalized to muscle surface area. $\boldsymbol{F}$, Quantification of average bouton area. $\boldsymbol{G}$, Quantification of total presynaptic area normalized to muscle surface area. $\boldsymbol{H}, \boldsymbol{I}$, UAS-RFP-tagged Dsmu1 $(\boldsymbol{H})$ expressed in a subset of motor neurons with 0K319-Gal4 colocalizes with the neural nuclear protein Elav in the ventral nerve cord and is also present in the cytoplasm (I, Dsmu1 red, Elav green). Scale bar: (in $\boldsymbol{H}) \boldsymbol{H}, \boldsymbol{I}, 10 \mu \mathrm{m}$. Error bars indicate SEM. ${ }^{*} p<0.05,{ }^{* *} p<0.01,{ }^{* * *} p<$ 0.001 , significance calculated versus wild-type control except where indicated.

ponent of Drosophila spliceosomes (Herold et al., 2009) and we renamed CG5451 dsmu1.

We identified a piggyBac transposon, pBacf03090 (Thibault et al., 2004), inserted between the first two protein-coding exons in the $d s m u 1$ gene. $d s m u 1^{p B a f f 03090} / \mathrm{Df}(3 \mathrm{R})$ Exel6182 mutants had a comparable synaptic phenotype to beag mutants (Fig. $2 B, C$ ). dsmu1 mutant NMJs had a $30 \%(p<0.001)$ decrease in synaptic bouton number (Fig. $2 E)$ and a $77 \%(p<0.001)$ increase in average synaptic bouton area (Fig. $2 F$ ), leading to a $29 \%$ increase in total synaptic area $(p<0.05)$ (Fig. $2 G)$. To determine the subcellular localization of Dsmul, we generated an epitope-tagged, Gal4/UASdriven Dsmul cDNA transgene. Expression of this transgene in a subset of motor neurons with OK319-Gal4 demonstrated that Dsmu1, like Beag, colocalized with Elav in the nucleus; however, we also observed some Dsmul protein in the cytoplasm (Fig. $2 \mathrm{H}, \mathrm{I}$ ).
Mammalian SMU1 protein has also been found to localize both to the nucleus and cytoplasm (Di Benedetto et al., 2001).

To test if beag and dsmul function in the same genetic pathway, we first looked for a genetic interaction between beag and dsmu1 heterozygotes. beag, dsmu1 transheterozygotes had an $11 \%$ decrease ( $p<$ $0.05)$ in bouton number compared with dsmu1 heterozygotes and a $14 \%$ decrease $(p<0.05)$ compared with beag heterozygotes (Fig. $2 E$ ), suggesting the two genes function together. To test this further we generated beag, dsmul double mutants. The decrease in the synaptic bouton number of these mutants was not significantly different from that observed in single mutants of beag or dsmu1 (Fig. 2D,E).Together, these data demonstrate that both genes are required for normal synaptic development in Drosophila and suggest that, similar to their homologs in C. elegans, these two genes function together.

Beag and Dsmul function in neurons to regulate NMJ growth

To determine in which cells Beag and Dsmul are required for normal NMJ synapse development, we constructed transgenes encoding the beag or dsmul ORF under Gal4/UAS control (Brand and Perrimon, 1993). Restoration of Beag or Dsmul expression with the musclespecific driver G14-Gal4 (Aberle et al., 2002) did not rescue the synaptic bouton number of the respective mutants (data not shown). In contrast, when driven with the motor neuron driver OK6-Gal4 (Aberle et al., 2002) (Fig. 3C,H) or the panneural driver C155-Gal4 (Lin and Goodman, 1994) (Fig. 3F, H), UAS-Beag and UAS-Dsmul fully rescued the decreased bouton number observed in beag and $d s m u 1$ mutants, respectively. Neural expression of epitope-tagged UAS-Beag and UAS-Dsmul also fully rescued the decreased bouton number in beag and dsmu1 mutants, respectively (data not shown). Neural overexpression of Beag or Dsmu1 in a wild-type background had no effect on the synaptic bouton number or area (Fig. $3 H$ ). Expression of transgenic Beag in all neural cells with C155-Gal4 or solely in glutamatergic neurons (which include motor neurons) with OK371-Gal4 (Mahr and Aberle, 2006) also rescued beag mutant adult viability (data not shown). Therefore, both Beag and Dsmul are required in neural cells for normal NMJ development, and even though Beag is broadly expressed, it is predominantly required in neurons for adult viability.

We also used these transgenes to further probe the genetic interactions between beag and dsmul. Neural overexpression of Dsmul with C155-Gal4 did not alter the bouton number of beag mutants (Fig. $3 D, H$ ). In contrast, neural overexpression of Beag resulted in a $28 \%(p<0.01)$ increase in bouton number of $d s m u 1$ mutants (Fig. $3 G, H$ ). These results confirm that beag and $d s m u 1$ 
are components of a common genetic pathway and indicate that beag functions downstream of $d s m u 1$.

\section{Beag and Dsmu1 are required for normal neurotransmitter release at the NMJ}

To determine the functional consequences of beag and dsmu1 mutations, we examined neurotransmitter release properties at the larval NMJ. Beag $g^{1 / E P 3260} \mathrm{mu}-$ tants had a $47 \%$ decrease $(p<0.001)$ in EJP amplitude compared with wild type (Fig. 4A-C). Similarly, dsmu1 mutants had a $43 \%$ decrease $(p<0.001)$ in EJP amplitude compared with wild type (Fig. 4C). mEJP amplitude and frequency were unchanged in beag ${ }^{1 / E P 3260}$ mutants leading to a $34 \%(p<0.001)$ decrease in quantal content compared with wild type (Fig. 4A, B,D-F). The decrease in quantal content observed in beag mutants suggested that the defect in EJP amplitude in these mutants was presynaptic in origin. This was confirmed when we fully rescued the reduction in EJP amplitude and quantal content in beag mutants by expressing UAS-Beag in motor neurons with OK6Gal4 (Fig. 4C-F). To determine whether the decrease in EJP amplitude observed in beag mutants could be due to a reduced number of neurotransmitter release sites, we counted the number of active zones labeled by Bruchpilot (Kittel et al., 2006; Wagh et al., 2006) in wild-type and beag mutant terminals. We found that beag mutant NMJ terminals had no difference in the number of active zones labeled with NC82 compared with wild type (Fig. 4GI). In addition, measurement of EJP amplitude in wild-type and beag mutant larvae at a range of extracellular calcium concentrations revealed that at high calcium concentrations the difference in EJP amplitude was exaggerated in beag mutants. This is consistent with a defect in calcium regulation of neurotransmitter release (data not shown). Our results therefore suggest that both Beag and Dsmul are required for normal neurotransmitter release as well as for morphological development of the NMJ.
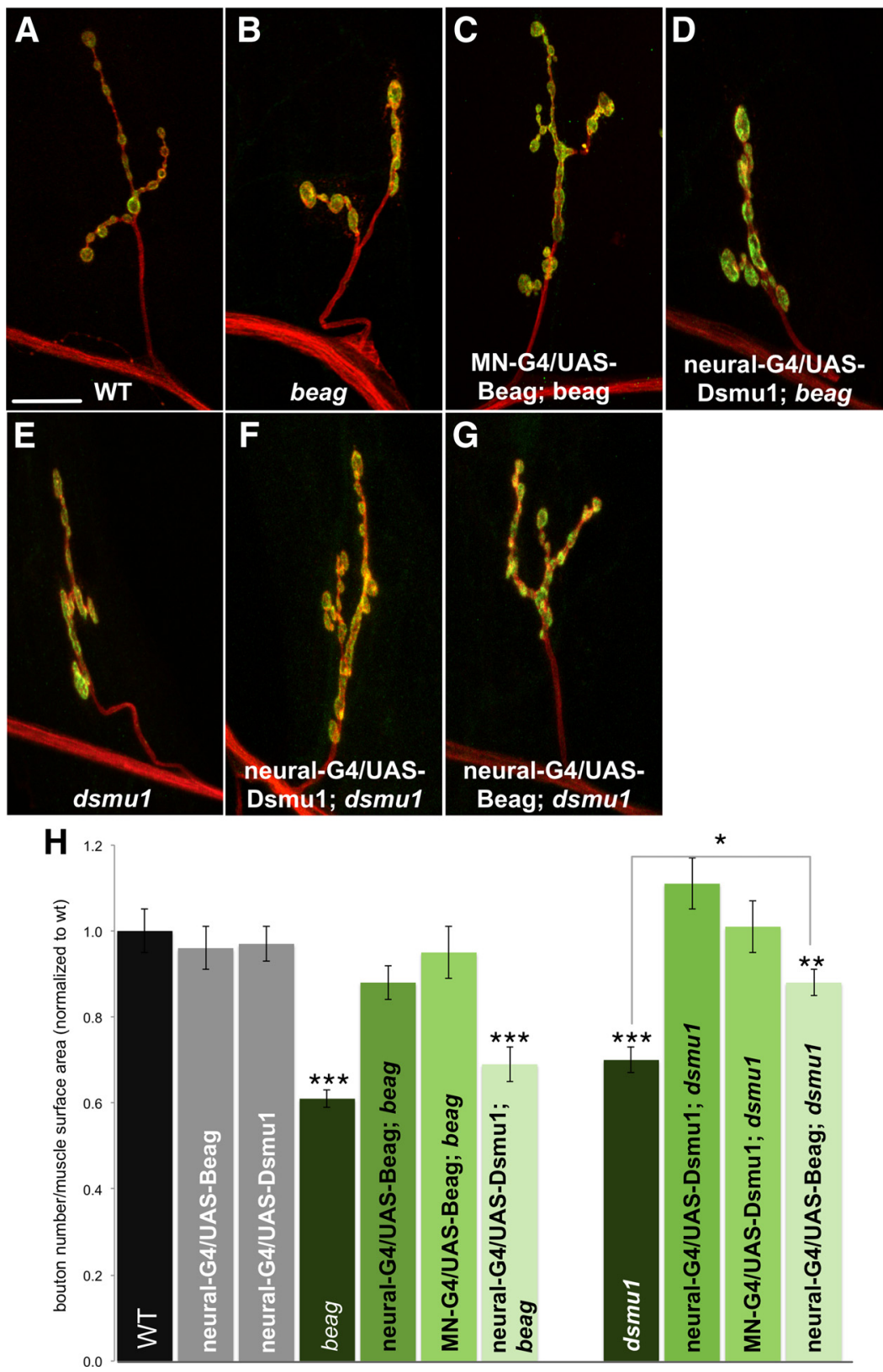

Figure 3. Beag and Dsmu1 are required in neurons for normal NMJ morphology. Expression of transgenic Beag in motor neurons with 0K6-Gal4 $(\boldsymbol{C})$ or all neural cells with C155-Gal4 (D) in beag ${ }^{1} / \mathrm{Df}(\boldsymbol{B})$ mutants rescues synaptic bouton number to wild-type levels $(\boldsymbol{A})$. Expression of transgenic Dsmu1 in motor neurons with 0K6-Gal4 $(\boldsymbol{H})$ or in neural cells with C155-Gal4 $(\boldsymbol{F})$ rescues the decrease in synaptic bouton number in $d s m u 7^{p B a c 03090} / \mathrm{Df}$ mutants $(\boldsymbol{E})$ to wild-type levels. Neuronal expression of Dsmu1 with C155-Gal4 in beag ${ }^{7}$ Df mutants $(\boldsymbol{H})$ does not rescue synaptic bouton number, while neural expression of Beag with (155-Gal4 in dsmu $1^{p B a c f 03090} \mathrm{Df}$ mutants $(\boldsymbol{G})$ partially rescues bouton number. Quantification of synaptic bouton numbers divided by muscle surface area $(\boldsymbol{H})$. Representative images are NMJ synaptic terminals at muscle 4 of segment A3 stained with anti-CSP (green) to label the presynapse and anti-HRP (red) to label the neuronal membrane. Error bars indicate SEM. ${ }^{*} p<0.05$, ${ }^{* *} p<$ $0.01,{ }^{* * *} p<0.001$, significance calculated versus wild-type control except where indicated. Scale bar, $20 \mu \mathrm{m}$.

\section{Synaptic FasII expression is altered in an isoform-specific manner in beag \\ mutants}

We next sought to determine whether altered splicing of specific genes could explain the observed beag NMJ phenotypes. Both the C. elegans and mammalian homologs of Beag have been shown to regulate alternative splicing of homologs of perlecan (encoded by unc-52 in C. elegans and trol in Drosophila) (Lundquist and Herman, 1994; Park et al., 2003; Spartz et al., 2004; Sugaya et al., 2006). We investigated if Beag could regulate NMJ development and function via modulation of perlecan; however, neither immunohistochemical nor genetic data supported an interaction between perlecan and Beag in the regulation of NMJ synaptic development (data not shown). Therefore, to identify synaptic molecules that could be regulated by beag and $d s m u 1$, we used antibodies against protein components of the presynaptic terminal to look for changes in expression level or localization in beag mutants. For the majority of antibodies we tested, including antibodies against CSP (Zinsmaier et al., 1990), Bruchpilot (Wagh et al., 2006), DAP160 (Marie et al., 2004), Dlg (Parnas et al., 

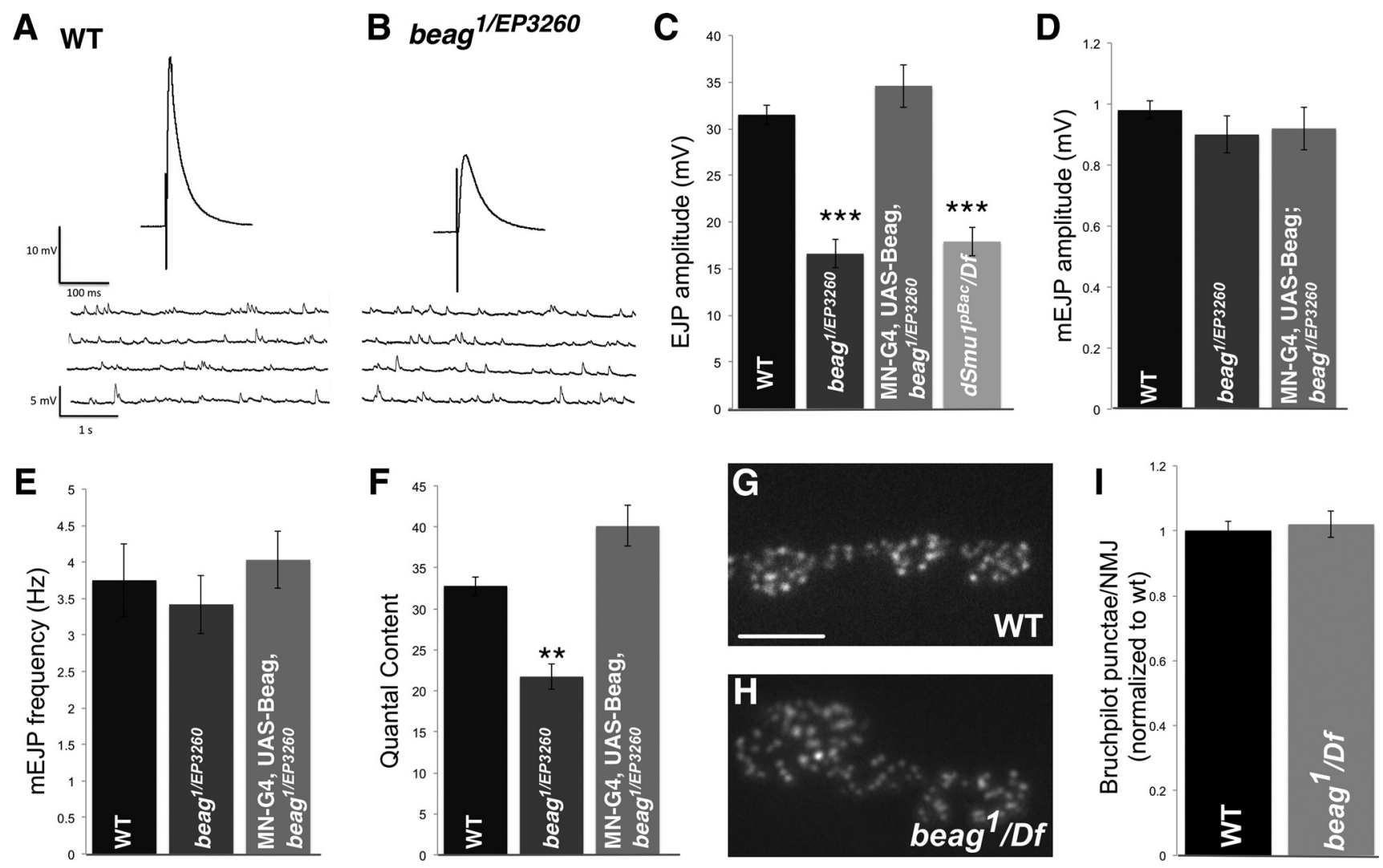

Figure 4. beag mutants have decreased neurotransmitter release. $A, B$, Representative traces recorded from muscle 6 of segment $A 3$ in wild-type and beag ${ }^{1 / E P 3260}$ larvae. beag $g^{1 / E P 3260}$ mutants have decreased EJP amplitude $(\boldsymbol{C})$ but normal $\mathrm{mEJP}$ amplitude $(\boldsymbol{D})$ and frequency $(\boldsymbol{E})$. This results in a decrease in quantal content in beag mutants $(\boldsymbol{F})$. Expression of transgenic Beag in motor neurons with OK6-Gal4 in Beag ${ }^{1 / E P 3260}$ mutants restores EJP amplitude $(\boldsymbol{C})$ and quantal content $(\boldsymbol{F})$.dsmu $7^{p B a c f 03090} / \mathrm{Df}$ mutants also have decreased EJP amplitude $(\boldsymbol{C})$, similar to beag mutants. The number of active zones labeled by anti-Bruchpilot is unchanged in beag $/$ Df mutants $(\boldsymbol{H})$ compared to wild type (G). Quantification of Bruchpilot punctae per NMJ terminal $(\boldsymbol{I})$. Error bars indicate SEM. ${ }^{* *} p<$ $0.01,{ }^{* * *} p<0.001$, significance calculated versus wild-type control.

2001), Map-1B (Fujita et al., 1982), Neuroglian (Hortsch et al., 1990), Phospho-Mad (McCabe et al., 2004), Synaptotagmin (Dubuque et al., 2001), VAP (Tsuda et al., 2008), and Wallenda (Collins et al., 2006), we did not observe any changes in expression level or localization. However, we did note a significant decrease in levels of synaptic FasII in beag mutants compared with wild type using the isoform-specific anti-FasII monoclonal antibody 1D4 (Fig. 5A,B,D) (Van Vactor et al., 1993).

The fasII gene generates four known isoforms, FasII-APEST +, FasII-A-PEST - , FasII-B, and FasII-C, generated by alternative splicing of several exons at the $3^{\prime}$ end of the fasII gene (Fig. 5A; see Materials and Methods for other names that have been used to refer to these isoforms) (Grenningloh et al., 1991; Lin and Goodman, 1994). All four isoforms are identical in the 737 aa at the $\mathrm{N}$ terminal, which forms the majority of the extracellular domain of the protein. FasII-A-PEST + and FasII-APEST - encode transmembrane proteins that have identical extracellular and intracellular domains with the exception of 29 aa encoded by a single exon that is included in FasII-APEST + but excluded in FasII-A-PEST - . The FasII-B isoform is poorly characterized. The FasII-C isoform has no cytoplasmic domain and has been suggested to attach to the membrane through a GPI linkage (Grenningloh et al., 1991). The functional differences between these four isoforms have not been systematically studied.

The anti-FasII 1D4 monoclonal antibody recognizes the intracellular domains of the FasII-A-PEST + and FasII-A-PESTisoforms, but does not recognize FasII-C and likely does not rec- ognize FasII-B (Van Vactor et al., 1993; Lin and Goodman, 1994; Schuster et al., 1996a). To determine the levels of synaptic and axonal FasII we used confocal microscopy and MetaMorph software. We measured anti-FasII staining intensity within the area that was costained with anti-HRP antibody, a specific marker of insect neuronal membranes (Jan and Jan, 1982). Intensity of NMJ staining with 1D4 was reduced by $26 \%$ in beag mutants compared with wild type $(p<0.001)$ and the intensity of motor neuron axon staining was decreased by $12 \%(p<0.05)$ (Fig. $5 B, D)$. We did not, however, observe any change in the distribution of FasII within NMJ synaptic boutons in beag mutants (data not shown). The reduction in synaptic 1D4 staining in beag mutants was fully rescued by the Beag genomic transgene (Fig. 5D). Similar to beag mutants, the intensity of synaptic 1D4 staining was decreased in dsmu1 mutants by $42 \%$ compared with wild type $(p<0.001)$ and motor neuron axon staining was reduced by $13 \%(p<0.01)$ (Fig. 5D). In contrast, when we used a different anti-FasII monoclonal antibody, 34B3, which recognizes the extracellular domain of FasII common to all four isoforms (Grenningloh et al., 1991), we observed no difference in synaptic staining intensity in beag or dsmu1 mutants compared with controls (Fig. 5C,D). We did, however, observe a 19\% increase $(p<$ 0.001 ) in 34B3 staining in the motor neuron axons of beag mutants (Fig. 5C). The alterations in FasII levels appear to be motor neuron specific, as we did not observe changes in FasII protein levels in other neurons in the CNS (data not shown). Together, our results reveal that levels of transmembrane FasII are reduced at the NMJ and in motor neuron axons in beag and 
dsmu1 mutants, whereas axonal levels of other isoforms of FasII are increased.

To determine whether the alteration in FasII protein isoform levels was paralleled by an isoform-specific change in FasII mRNA levels, we performed quantitative real-time PCR (qRT-PCR) from cDNA isolated from wild-type or beag larval brains. We found that the mRNA level of the combined FasII-A-PEST + and FasIIA-PEST - isoforms was decreased in beag mutants by $32 \%$ compared with wild type $(\mathrm{SE}=9)$ (Fig. $5 E)$. In contrast, the FasII-C mRNA level was increased by $17 \%$ compared with wild type by qRT-PCR (SE = 12) (Fig. 5E). Total FasII mRNA levels were not changed in beag mutants compared with wild type (Fig. 5E). To determine whether the levels of individual FasII-A-PEST + and PEST - isoforms were also altered in beag mutants we measured their relative levels by semiquantitative PCR (Fig. 5F,G). In wild-type animals we found that message for FasIIA-PEST + was more abundant than message for FasII-A-PEST-. In beag mutants, we found that this ratio was altered with a relative decrease $(-42 \%, p<0.05)$ in the ratio of FasII-A-PEST + to PEST - message. Therefore both immunohistochemistry and mRNA transcript analysis are consistent with a selective reduction of FasII-A-PEST + in beag mutants.

\section{fasII and beag function in the same genetic pathway to regulate NMJ growth}

fasII loss-of-function mutants have previously been reported to have a decrease in bouton number similar to what we observe in beag mutants (Schuster et al., 1996a). To confirm this result and to determine whether fasII and beag function in the same genetic pathway, we examined the NMJ phenotype of three fasII alleles. fasII $^{\text {e76 }}$ is a hypomorphic allele that produces $\sim 10 \%$ of the wild-type level of FasII protein (Grenningloh et al., 1991). fasI$I^{e B 112}$ is a protein-null allele (Grenningloh et al., 1991), and Df(1)BSC869 is a deficiency that fully removes the fasII locus in addition to other genes. fasII ${ }^{\text {e76/e76 }}$ $(-50 \%, p<0.001)$, fasII e76/eB112 $^{\text {( }}(-50 \%$, $p<0.001)$, and fasII $I^{e 76} / \mathrm{Df}(-59 \%, p<$ $0.001)$ mutant combinations all had fewer synaptic boutons than controls (Fig. $6 F, H$ ). In contrast, heterozygotes of any of these alleles had no change in bouton number compared with controls (Fig. 6C,H), even though synaptic FasII levels were reduced (data not shown).

We then tested whether beag and fasII function in the same genetic pathway. Like fasII heterozygotes, beag heterozygotes had no decrease in bouton number compared with controls (Fig. $6 B, H)$. However, animals heterozygous for both $f_{a s I I}{ }^{e 76}$ and
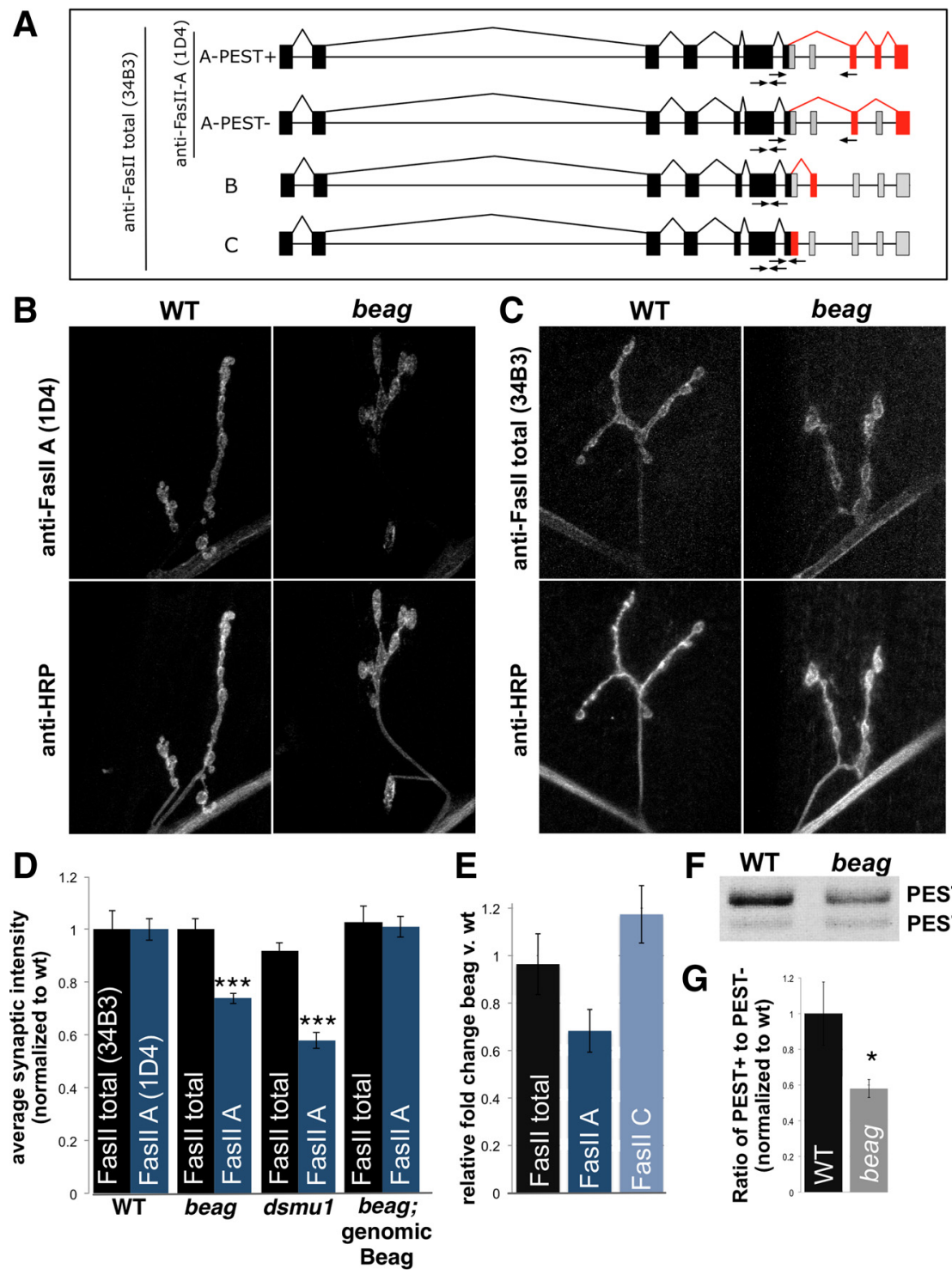

E

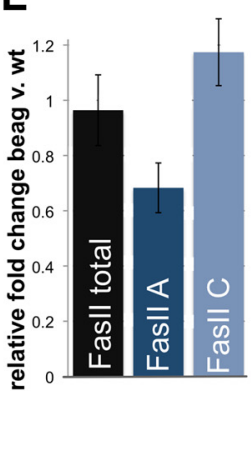

F WT beag

G

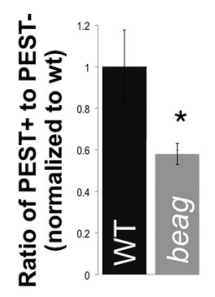

PEST+

Figure 5. Fasll levels are altered in an isoform-specific manner in beag mutants. $A$, Fasll has four splice isoforms, A-PEST+ , A-PEST - , B, and C, that all include the first seven exons (black) but differ in the inclusion (red) or exclusion (gray) of exons at the $3^{\prime}$ end of the gene. The monoclonal anti-Fasll total antibody (34B3) recognizes an epitope in the extracellular domain of all four isoforms. The monoclonal anti-Fasll-A antibody (1D4) recognizes an epitope in the intracellular domain of Fasll-A-PEST + and Fasll-A-PEST - . Positions of the primers used to amplify both Fasll-A isoforms, the C isoform, or total Fasll for qRT-PCR are indicated by arrows. $\boldsymbol{B}, \boldsymbol{C}$, NMJs at muscle 4 of segment A3 in third instar larvae were stained with the monoclonal antibody 1D4 or the monoclonal antibody 34B3. The level of $1 D 4$ staining in beag ${ }^{7} / D f$ mutant NMJs is reduced compared with wild type $(\boldsymbol{B})$. In contrast, there is no difference between wild-type and beag NMJs when stained with 34B3 (C). D, Quantification of relative staining intensity with 1D4 and 34B3 at wild-type, beag, and dsmu 1 mutant synapses. Expression of a genomic Beag transgene in beag ${ }^{1} / \mathrm{Df}$ mutants rescues synaptic Fasll-A staining intensity to wild-type levels. dsmu1 mutants also have decreased synaptic 1D4 staining. $\boldsymbol{E}$, qRT-PCR measurement of the relative abundance of both Fasll-A isoforms, Fasll-C, and total Fasll. Fasll-A mRNA is decreased in beag mutants, while Fasll-C mRNA is slightly increased. No change was observed in total Fasll mRNA abundance in beag mutants. $\boldsymbol{F}$, Measurement of Fasll-A-PEST + and Fasll-A-PEST - mRNA levels by semiquantitative multiplex PCR in wild-type and beag brains demonstrates a decrease in the ratio of FasIl-A-PEST + to Fasll-A-PEST - in beag mutants. G, Normalized ratio of FasIl-APEST + versus Fasll-A-PEST - in wild-type and beag mutants. Error bars indicate SEM. ${ }^{*} p<0.05,{ }^{* * *} p<0.001$, significance calculated versus wild-type control.

beag $^{1}$ had a $42 \%$ decrease in synaptic bouton number $(p<0.001)$ compared with wild type (Fig. $6 D, H$ ). This decrease was not significantly different from the decrease in bouton number observed in beag $^{1} /$ Df larvae or in fasII ${ }^{76 / e 76}$ larvae (Fig. 6E, F,H). Furthermore, combining beag $^{1} / \mathrm{Df}$ mutants with heterozygous or homozygous $\mathrm{fasI}^{\mathrm{e} / 6}$ did not further decrease bouton number compared with beag mutants alone (Fig. $6 E-H$ ). These data suggest a strong genetic interaction between fasII and beag. 

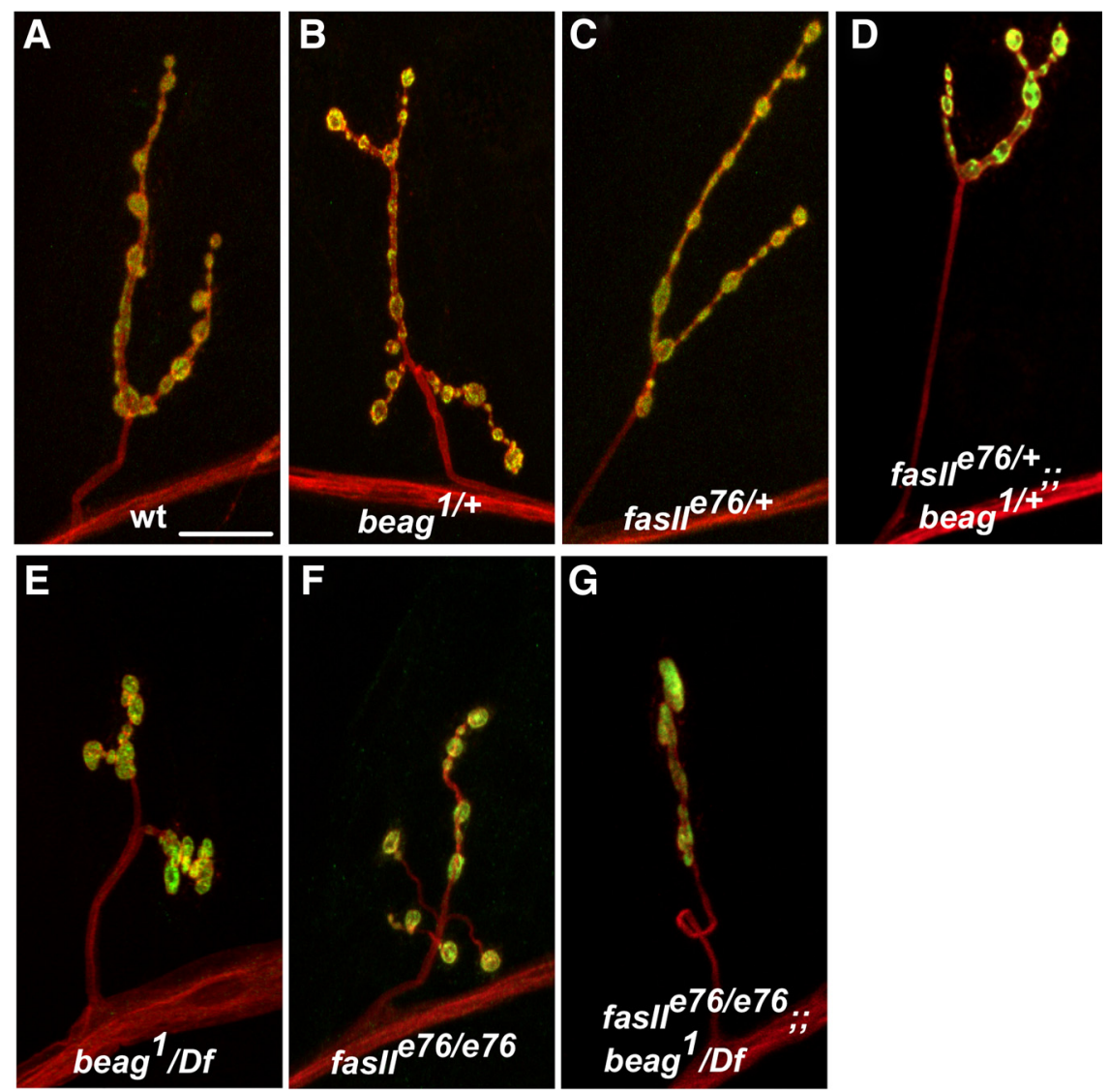

H
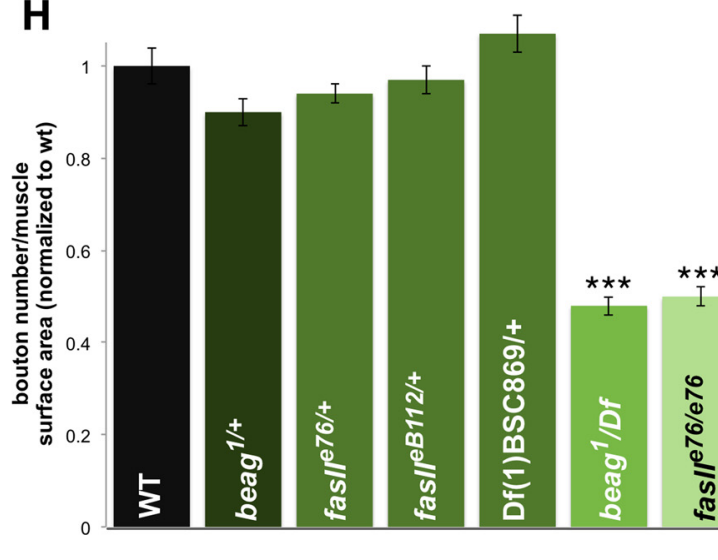

Figure 6. fasll and beag genetically interact. Neither beag ${ }^{1 /+}$ heterozygotes nor fas// ${ }^{76 /+}$ heterozygotes have a significant change in bouton number compared with wild-type $(\boldsymbol{A}, \boldsymbol{B}, \boldsymbol{C}, \boldsymbol{H})$; however, beag ${ }^{1 /+}$, fass $^{\mathrm{p}{ }^{76 /+}+}$ trans-heterozygotes havefewerboutons than controls $(\boldsymbol{D}, \boldsymbol{H})$. Removal

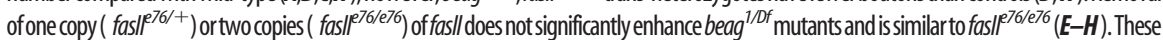
results demonstrate that fasll and beag are components of a common genetic pathway in the regulation of NMJ development. $\boldsymbol{H}$, Quantification of bouton number normalized to muscle surfacearea. Representativeimages NMJ synapticterminals at muscle 4 of segment $A 3$ stained with anti-CSP (green) to label the presynapse and anti-HRP (red) to label the neuronal membrane. Error bars indicate SEM. ${ }^{* * *} p<0.001$, significance calculated versus wild-type control. Scale bar, $20 \mu \mathrm{m}$.
The transmembrane isoforms of FasII are necessary and sufficient for normal NMJ growth

Our data suggested that the beag phenotype was linked to misregulation of FasII isoforms. We therefore set out to determine which isoforms of FasII are required for normal NMJ growth. To do this we used an RNAi transgene that targets all isoforms of FasII (FasIIRNAi-Total; Dietzl et al., 2007) and we also generated an RNAi transgene that targets only the transmembrane FasII isoforms (FasIIRNAi-A). Ubiquitous expression of either of these RNAi constructs with Da-G4 caused a large decrease in FasII protein levels both at the NMJ and in the brain (data not shown). Knock- down of all FasII isoforms with FasIIRNAiTotal resulted in a 32\% $(p<0.001)$ decrease in synaptic bouton number compared with controls (Fig. $7 B, K$ ). Knockdown of the transmembrane isoforms of FasII with FasIIRNAi-A resulted in a $20 \%(p<0.05)$ decrease in bouton number (Fig. $7 C, K$ ), demonstrating that the transmembrane isoforms of FasII are required for normal NMJ growth. To determine whether FasII$\mathrm{A}-\mathrm{PEST}+$, FasII-A-PEST-, or both were required for normal NMJ growth, we generated RNAi-resistant FasII-A-PEST + and $\mathrm{PEST}-$ transgenes. In these transgenes, the portion of the wild-type cDNA targeted by FasIIRNAi-A was replaced with a synthetic sequence in which codon usage was changed such that the amino acid sequence was maintained but the mRNA would not be degraded by FasIIRNAi-A. Ubiquitous expression of RNAi-resistant FasII-APEST + or RNAi-resistant FasII-A-PESTfully rescued the reduction in bouton number induced by FasIIRNAi-A expression (Fig. $7 E, F, K$ ). In contrast, expression of FasII-C did not lead to a significant increase in bouton number in larvae in which the transmembrane FasII isoforms were knocked down (Fig. 7D,K). These results indicate that transmembrane FasII is required for normal NMJ growth.

We next tested which isoforms of FasII were sufficient to rescue NMJ growth in fasII mutants. Using the $34 \mathrm{~B} 3$ antibody, which detects all FasII isoforms, we determined that each transgene expressed comparable levels of protein (data not shown). We observed increased levels of FasII in neuronal soma, axons, and NMJ synapses when FasII-A-PEST + and FasII-A-PESTwere overexpressed. In contrast, while overexpression of FasII-C led to a similar increase in neuronal soma FasII levels, there was a smaller increase in synaptic FasII levels at the NMJ compared with overexpression of FasII-A-PEST + or FasII-A-PEST - (data not shown). Expression of FasII-C in motor neurons of fasII $I^{e 76} / \mathrm{Df}$ mutants with OK6Gal4 did not rescue bouton number (Fig. $7 \mathrm{H}, \mathrm{K})$. In contrast, expression of FasII-APEST + or FasII-A-PEST - fully rescued bouton number in fasII mutants to wildtype levels (Fig. 7I-K). Therefore, both RNAi and rescue experiments demonstrate that the transmembrane isoforms of FasII are essential for normal NMJ development, in contrast to the FasII-C isoform.

\section{FasII-A-PEST +, but not other FasII isoforms, rescues beag NMJ morphological defects}

Transmembrane isoforms of FasII are reduced in beag mutants and we have shown that these isoforms are essential for normal NMJ development. We therefore attempted to rescue beag mutants by expressing cDNA transgenes (which lack introns) 

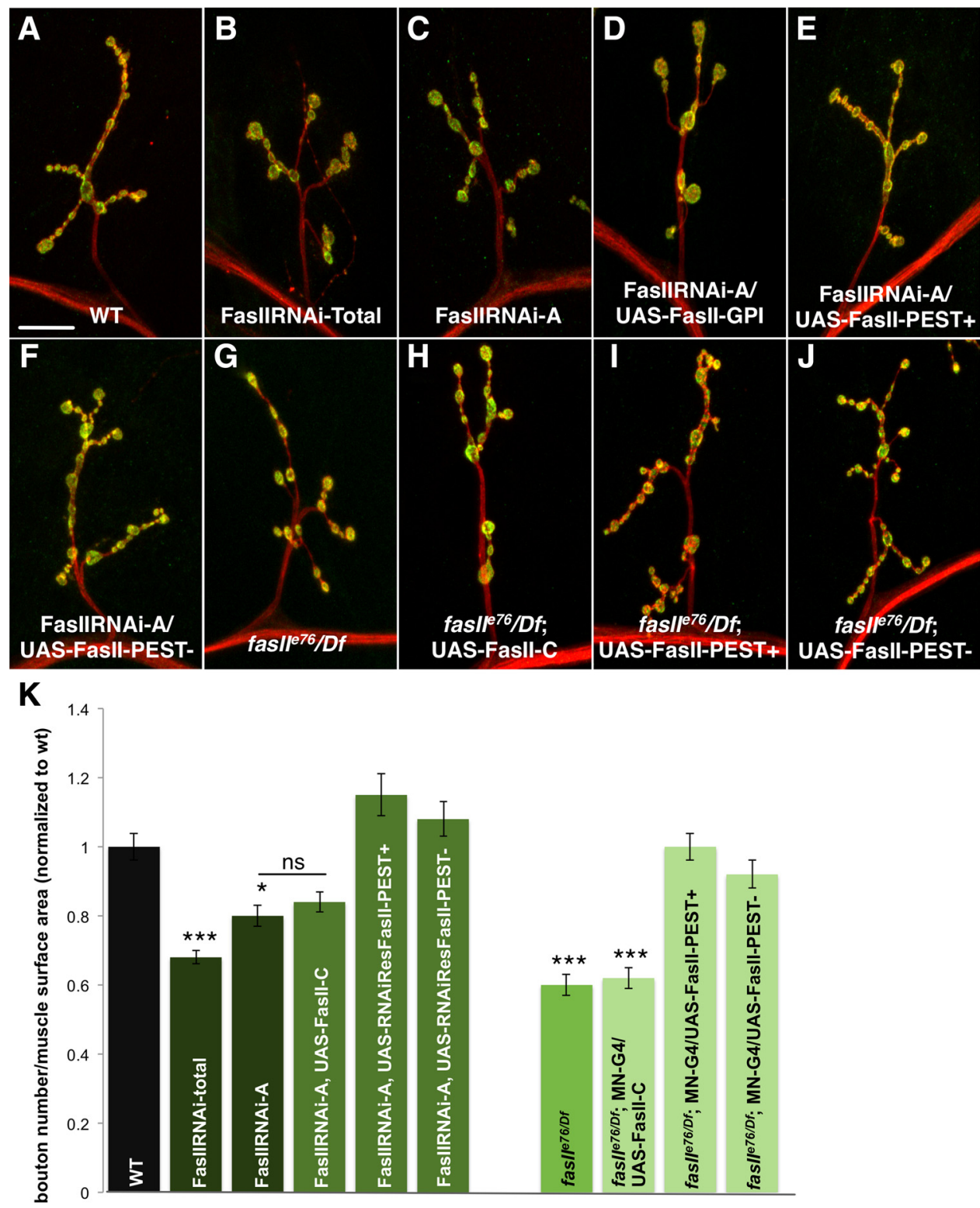

Figure 7. Fasll-A-PEST + and Fasll-A-PEST - are necessary and sufficient for normal NMJ growth. Ubiquitous (Da-Gal4) RNAi-induced knockdown of all Fasll isoforms with UAS-FasIIIRNAi-Total $(\boldsymbol{B})$ or only Fasll-A-PEST + and FasIl-A-PEST - with UAS-FasIIRNAi-A $(\boldsymbol{C})$ causes a decrease in synaptic bouton number compared with wild type $(\boldsymbol{A})$. The decrease in synaptic bouton number due to RNAi inhibition of transmembrane Fasll isoforms is not altered by coexpression of Fasll-C (D), but is rescued by coexpression of RNAi resistant forms of Fasll-A-PEST + (E) or Fas II-A-PEST - $(\boldsymbol{F})$. Similarly, the decrease in bouton number in fas $/\left.\right|^{76} /$ Df mutants $(\boldsymbol{G})$ is not rescued by expression of Fasll-C in motor neurons (OK6-Gal4) (H), while expression of Fasll-A-PEST + or Fasll-A-PEST fully restores the synaptic bouton number of these mutants to wild-type levels $(\boldsymbol{I}, \boldsymbol{J}) . \boldsymbol{K}$, Quantification of synaptic bouton number normalized to muscle surface area. Representative images of NMJ synaptic terminals at muscle 4 of segment A3 stained with anti-CSP (green) to label the presynapse and anti-HRP (red) to label the neuronal membrane. Error bars indicate SEM. ${ }^{*} p<0.05$, ${ }^{* * *} p<$ 0.001 , significance calculated versus wild-type control. Scale bar, $20 \mu \mathrm{m}$.

encoding FasII-A-PEST+, FasII-A-PEST-, or FasII-C. Neural overexpression of FasII-A-PEST+ or FasII-C had no effect on NMJ synaptic bouton number (Fig. 8G). However, overexpression of FasII-A-PEST - did induce a significant increase in synaptic bouton number (Fig. $8 G$ ). We then tested the ability of these transgenes to rescue beag mutants. Neither FasII-A-PEST - nor FasII-C expression altered beag mutant NMJ morphology (Fig. $8 C, D, G)$. In contrast, expression of FasII-A-PEST + fully rescued beag mutant synaptic bouton number and average synaptic bouton area to wild-type levels (Fig. $8 E, G, H$ ). These rescue experiments demonstrate a difference between FasII-A-PEST+ and FasII-A-PEST - function at the NMJ. In addition, these results, combined with FasII protein and mRNA measurements, suggest that a specific reduction in the FasII-A-PEST+ isoform is suffi- cient to explain the synaptic morphological defects in beag mutants. We also found that neural expression of FasII-A-PEST+ partially rescued $d s m u 1$ mutant synaptic bouton number by $15 \%$ $(p<0.05)$ providing evidence that the decrease in bouton number in dsmu1 mutants is also at least in part due to a reduction in FasII-A-PEST + and further supporting a common role for Beag and Dsmul in the regulation of NMJ morphology through modulation of FasII.

While synaptic morphological development can be rescued by restoration of FasII-A-PEST+ in beag mutants, we wished to determine whether neurotransmitter release properties were also restored. We measured EJP amplitude in beag mutants expressing transgenic FasII-A-PEST + and found that the defect in evoked neurotransmitter release was not rescued. In addition, 

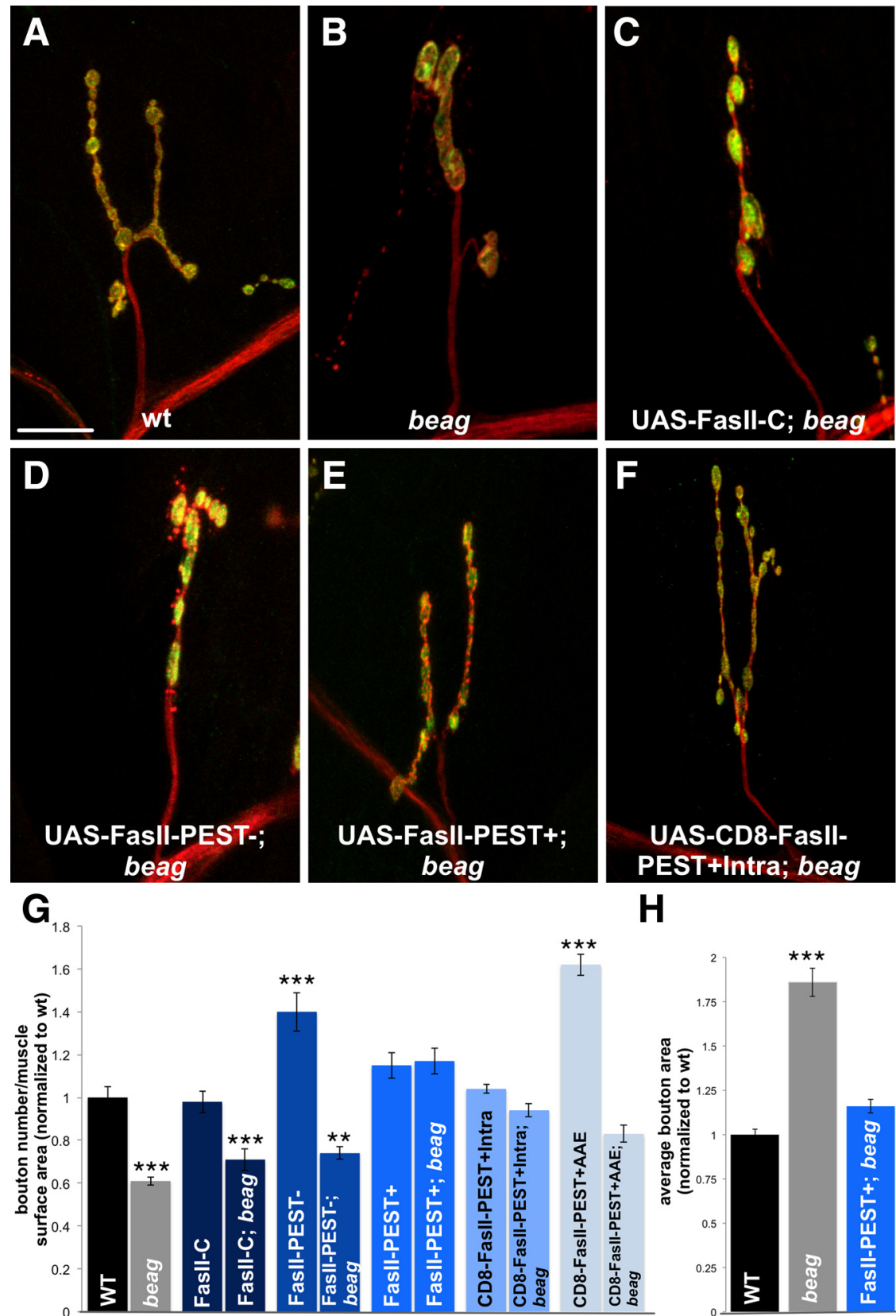

H

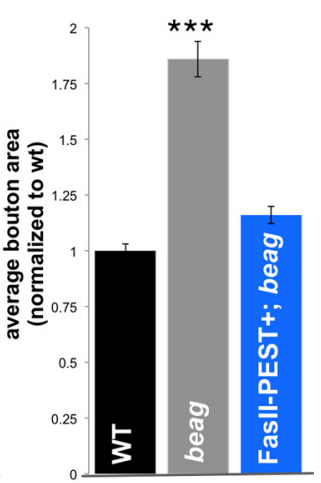

Figure 8. Overexpression of Fasll-A-PEST + but not the other Fasll isoforms rescues beag NMJ morphology. Expression of Fasll-A PEST+ or Fasll-C in motor neurons with 0K6-Gal4 in a wild-type background $(\boldsymbol{A})$ does not affect synaptic bouton number, while expression of Fasll-A-PEST - causes an increase in synaptic bouton number (G). Expression of Fasll-C ( $\boldsymbol{C}$ or Fasll-A-PEST - $(\boldsymbol{D})$ in motor neurons (OK6-Gal4) of beag ${ }^{1}$ Df mutants $(\boldsymbol{B})$ does not rescue synaptic bouton number, while expression of Fasll-A-PEST - in motor neurons restores normal synaptic bouton number in beag ${ }^{7} / D$ f mutants $(\boldsymbol{E})$. Expression of a fusion protein in which the extracellular and transmembrane domains of CD8 are fused to the intracellular domain of FasIl-A-PEST + (CD8-FasIl-A-PEST + Intra) in motor neurons with 0K6-Gal4 has no effect on synaptic bouton number in a wild-type background (G); however, expression in beag ${ }^{1}$ Df mutants fully restores synaptic bouton number $(\boldsymbol{F})$. Expression of Fasll-A-PEST + AAE in motor neurons with OK6-Gal4 in a wild-type background causes an increase in synaptic bouton number and expression in beag mutants rescues synaptic bouton number (G). Quantification of bouton number normalized to muscle surface area $(\boldsymbol{G})$. $\boldsymbol{H}$, Motor neuron expression of Fasll-A-PEST + with OK6-Gal4 also rescues the average bouton area of beag $^{7}$ /Df mutants. Representative images of NMJ synaptic terminals at muscle 4 of segment A3 stained with anti-CSP ( $\left.g r e e n\right)$ to label the presynapse and anti-HRP(red) to label the neuronal membrane. ErrorbarsindicateSEM. ${ }^{* *} p<0.01,{ }^{* * *} p<0.001$, significance calculated versus wild-type controls. Scale bar, $20 \mu \mathrm{m}$.

beag mutant viability was not rescued by neural expression of FasII-A-PEST + (data not shown). This result is consistent with previous studies showing that fasII can regulate synaptic structure without altering neurotransmitter release (Stewart et al., 1996).
The intracellular domain of FasII-APEST + is sufficient to rescue beag mutant synaptic morphology

Our data suggest that the intracellular domain of FasII, which is included in FasII$\mathrm{A}-\mathrm{PEST}+$ and FasII-A-PEST - , but not FasII-C, is important for FasII's synaptic function. We therefore examined if the extracellular domain of FasII was required to rescue beag mutants. To test this hypothesis we used a chimeric transgene in which the extracellular and transmembrane domains of FasII-A-PEST + were replaced with the extracellular and transmembrane domains of the human T-lymphocyte protein CD8 (Littman et al., 1985) fused in frame to the entire FasII-APEST + intracellular region (CD8-FasII-APEST+Intra) (Zito et al., 1997). When we drove expression of this construct in motor neurons, we saw a dramatic increase in synaptic FasII immunoreactivity using the 1D4 antibody and observed that CD8 was targeted to the synapse, demonstrating that the intracellular domain of FasII-A-PEST + is sufficient for presynaptic localization (data not shown). In wild-type larvae, expression of CD8-FasII-A-PEST + Intra did not affect synaptic bouton number (Fig. $8 G$ ), similar to expression of full-length FasII-A-PEST+. We then expressed this construct in the beag mutants. Similar to full-length FasII-APEST+, CD8-FasII-A-PEST+Intra fully rescued the decrease in synaptic bouton number in beag mutants to wild-type levels (Fig. $8 F, G$ ). This result demonstrates a specific requirement for the intracellular domain of transmembrane FasII in the rescue of synaptic morphology in beag mutants.

To further dissect the contribution of individual segments of the FasII-APEST + intracellular domain we expressed a version of FasII-A-PEST + in which the last three amino acids, which encode a PDZ-interaction domain, were altered from S-A-V to A-A-E (FasII-APEST + AAE). To determine the localization of this FasII transgene, we expressed it in the motor neurons of fasII $I^{e 76 / y} \mathrm{mu}-$ tants, which have no detectible synaptic or axonal FasII staining (data not shown). When FasII-A-PEST + AAE was expressed in these mutants, FasII staining was observed in both motor neuron axons and at the NMJ (data not shown). Thus, when overexpressed, the PDZ-interaction domain of FasII is not absolutely required for presynaptic localization of FasII. To determine whether the PDZ-interaction domain of FasII was required for rescue of beag mutant NMJ morphology, we then expressed FasII-A-PEST + AAE - in motor neurons of wild-type and beag mutants. Expression in wild-type animals led to an increase in bouton number, while expression in beag mutants re- 
stored bouton numbers to levels not significantly different from wild type (Fig. 8G). These results indicate that the PDZinteraction domain is not essential for the rescue of synaptic morphology in beag mutants by FasII.

\section{Discussion}

Regulation of the splice isoform diversity of synaptic adhesion molecules is essential for differential synaptic localization and function (Lisé and El-Husseini, 2006; Dalva et al., 2007). We have identified Beag as an alternative splicing factor that regulates specific isoforms of FasII, the Drosophila ortholog of NCAM. We establish that Beag works together with Dsmul in a neuronal genetic pathway required for NMJ synaptic terminal growth and neurotransmitter release. We show genetic interactions between fasII and beag and find a specific reduction of both mRNA and protein levels of transmembrane isoforms of FasII in beag mutants and similar protein changes in dsmul mutants. Through analysis of the requirements for individual FasII splice isoforms for normal NMJ growth, we find that the transmembrane isoforms of FasII are both necessary and sufficient for this process. We show that restoration of one of these transmembrane isoforms, FasII-A-PEST +, can completely rescue the synaptic structural defects of beag mutants while other FasII isoforms cannot, consistent with a relative reduction of FasII-A-PEST + transmembrane isoform mRNA levels in beag mutants. Finally, rescue of beag mutants by the FasII-A-PEST + intracellular region alone reveals a function in synapse development for this isoform independent of transsynaptic adhesion. Our data establish that Beag and Dsmul govern synaptic morphological development through the regulation of the alternative splicing of FasII.

\section{Beag and Dsmu1 function in the nervous system to regulate alternative splicing}

Beag, like SMU-2 and RED (Assier et al., 1999; Spartz et al., 2004), is expressed in many tissues. However, our rescue data demonstrate that Beag is required only in motor neurons for normal adult Drosophila viability, NMJ morphology, and neurotransmitter release. Neuronal sensitivity to the loss of ubiquitously expressed proteins has been demonstrated for other RNA regulatory proteins, notably the RNA processing factor Survival of Motor Neurons, the gene disrupted in spinal muscular atrophy (Monani, 2005). Potentially, Beag and Dsmul could interact with neuronal-specific proteins to regulate synapse development and function. Unlike most characterized alternative splicing factors, Beag and Dsmul proteins lack canonical RNA binding domains (although Dsmul does contain a WD domain which in Gemin 5 can bind RNA) (Lau et al., 2009) limiting biochemical techniques to identify pre-mRNA targets (Ule et al., 2005). It therefore seems likely that Beag and Dsmul exert their effects on pre-mRNA splicing through interactions with other RNA binding proteins similar to mechanisms described for splicing regulators such as Drosophila transformer and crooked neck (Tian and Maniatis, 1993; Edenfeld et al., 2006). Genetic model organisms are particularly useful for the identification and characterization of these types of factors. While we show that a change in FasII isoform distribution can explain the synaptic morphology defects in beag mutants, the neurotransmitter release and animal viability phenotypes of these mutants are not rescued by FasII expression. We hypothesize therefore that similar to other regulators of premRNA splicing, Beag and Dsmul alter the alternative splicing of multiple neuronal genes. It is also seems likely given their broad expression pattern that they may regulate some splicing events in non-neuronal tissues.

\section{Regulation of FasII alternative splicing by Beag}

Our data suggest that in beag mutants FasII alternative splicing is altered, resulting in a decrease in the level of the transmembrane FasII isoforms coupled with a change in the relative level of FasIIA-PEST + mRNA compared with FasII-A-PEST- mRNA. Splicing of Drosophila FasII transcripts generates at least four protein isoforms with identical extracellular domains but different forms of membrane attachment and intracellular domains (Grenningloh et al., 1991). This splicing pattern is conserved in both invertebrate and mammalian FasII homologs. In Manduca sexta, GPIlinked and transmembrane FasII isoforms are expressed by different cells and may have unique roles in cell migration, neurite outgrowth, and synapse formation (Wright et al., 1999, 2001; Knittel et al., 2001). In Aplysia, apCAM isoform expression also varies by cell type, and presynaptic and postsynaptic compartments can contain different apCAM isoforms (Mayford et al., 1992; Schacher et al., 2000). In mammals, the NCAM transmembrane isoform with the longest cytoplasmic domain, NCAM 180, is upregulated over the course of development and predominantly localizes to mature neuronal synapses, while the transmembrane isoform with a shorter cytoplasmic domain, NCAM 140 , is localized to growing axons and glia in addition to synapses (Cunningham et al., 1987; Barbas et al., 1988). In contrast, the GPI-linked isoform, NCAM 120, is expressed mainly in glia (Pollerberg et al., 1985, 1986; Persohn et al., 1989). Depolarization of cultured hippocampal neurons causes increased skipping of the NCAM 180-specific exon (Schor et al., 2009) and neural activity can also regulate the relative levels of apCAM isoforms (Schacher et al., 2000). Given the general conservation of splice isoform structure between these genes and FasII, it is possible that homologs of Beag and Dsmul could contribute to this regulation.

\section{FasII at the synapse}

Although important roles of FasII in synapse growth and plasticity are well established (Packard et al., 2003; Kristiansen and Hortsch, 2010), the expression and function of individual Drosophila FasII splice isoforms had not previously been systematically analyzed. In this study, we demonstrate that the transmembrane isoforms of FasII are essential for synaptic development. In contrast, FasII-C cannot rescue synaptic development in fasII mutants. These results are consistent with prior results showing that expression of transgenic FasII-A-PEST + , in contrast to FasII-C, can alter synapse function in the CNS (Baines et al., 2002). Previous studies have also shown that reducing levels of all FasII isoforms by $90-100 \%$ causes a large decrease in NMJ bouton number, which we confirm here (Schuster et al., 1996a, Ashley et al., 2005). However studies of more modest reductions of fasII in heterozygote mutants have been inconsistent (Schuster et al., 1996a, Ashley et al., 2005). Here we find that the bouton number is unchanged compared with wild type in larvae heterozygous for either of two fasII alleles or for a deficiency that removes the entire fasII gene. We have confirmed that these fasII manipulations have a $\sim 50 \%$ decrease in synaptic FasII levels. The contrast between fasII heterozygotes and beag mutants suggests that a change in the relative FasII isoform levels and the presynaptic to postsynaptic FasII ratio, rather than a uniform reduction in FasII levels, can produce aberrant synaptic morphology.

The importance for normal NMJ development of the relative levels and presynaptic and postsynaptic distribution of individual FasII isoforms is supported by several lines of evidence. Simultaneous presynaptic and postsynaptic overexpression of FasII-APEST + induces synaptic overgrowth (Ashley et al., 2005), while in contrast we find that solely presynaptic overexpression of 
FasII-A-PEST + does not alter NMJ morphology. In addition, we show that neuronal overexpression of FasII-A-PEST - can induce synaptic overgrowth while overexpression of FasII-C, like FasII-A-PEST+, does not, revealing unique effects of each isoform on synapse growth. Furthermore, expression of FasII-APEST + but not FasII-A-PEST - can rescue the beag NMJ morphology defects. These data suggest that these two isoforms have distinct activities and that a disruption in the presynaptic balance of transmembrane FasII isoforms at the NMJ in beag mutants leads to a decrease in bouton number, even while overall total synaptic FasII levels are maintained.

Our results also show that the intracellular domain of FasIIA-PEST + alone is sufficient to rescue beag mutant synapse morphology defects, revealing an important function for this domain that is independent of transynaptic adhesion. It is noteworthy that differences have been described in the downstream signaling of transmembrane isoforms of mammalian NCAM. For example, the src family kinase Fyn interacts with NCAM 140, but not 180, and this interaction is essential for neurite outgrowth (Beggs et al., 1994, 1997). In contrast, the scaffolding protein Spectrin has a higher affinity for NCAM 180 than 140, which is required for NCAM-induced recruitment of synaptic proteins to the postsynaptic density (Pollerberg et al., 1986, 1987; Leshchyns'ka et al., 2003). Little is known about the cytoplasmic signaling functions of either of the Drosophila FasII transmembrane isoforms. Comparison of NCAM 180 and 140 with FasII-A-PEST + and PESTshows little homology between their intracellular domains. The full cytoplasmic domain of FasII-A-PEST+ is sufficient for postsynaptic localization at the NMJ (Zito et al., 1997), and we show that this domain is also sufficient for NMJ presynaptic localization. The cytoplasmic domains of both FasII-A isoforms contain a C-terminal PDZ-binding sequence. We find this domain is not absolutely required for presynaptic localization of FasII-A$\mathrm{PEST}+$ and is also not required for rescue of beag mutants by FasII-A-PEST + . The only difference between FasII-A-PEST + and FasII-A-PEST - is one exon encoding 29 aa within the intracellular region. These 29 aa contain a PEST sequence (rich in proline, glutamic acid, serine, and threonine residues) that could preferentially target FasII-A-PEST + for proteasomal degradation (Rechsteiner, 1988). It is difficult, however, to relate enhanced degradation to the specific synaptic activity of FasII-APEST + in beag mutants. Nonetheless, analysis of beag and dsmu1 mutants has revealed unique roles for transmembrane FasII isoforms at the synapse and an important future goal will be to determine the nature of these distinctions.

\section{References}

Aberle H, Haghighi AP, Fetter RD, McCabe BD, Magalhães TR, Goodman CS (2002) wishful thinking encodes a BMP type II receptor that regulates synaptic growth in Drosophila. Neuron 33:545-558.

Ashley J, Packard M, Ataman B, Budnik V (2005) Fasciclin II signals new synapse formation through amyloid precursor protein and the scaffolding protein dX11/Mint. J Neurosci 25:5943-5955.

Assier E, Bouzinba-Segard H, Stolzenberg MC, Stephens R, Bardos J, Freemont P, Charron D, Trowsdale J, Rich T (1999) Isolation, sequencing and expression of RED, a novel human gene encoding an acidic-basic dipeptide repeat. Gene 230:145-154.

Baines RA, Seugnet L, Thompson A, Salvaterra PM, Bate M (2002) Regulation of synaptic connectivity: levels of Fasciclin II influence synaptic growth in the Drosophila CNS. J Neurosci 22:6587-6595.

Barbas JA, Chaix JC, Steinmetz M, Goridis C (1988) Differential splicing and alternative polyadenylation generates distinct NCAM transcripts and proteins in the mouse. EMBO J 7:625-632.

Beggs HE, Soriano P, Maness PF (1994) NCAM-dependent neurite outgrowth is inhibited in neurons from Fyn-minus mice. J Cell Biol 127:825-833.
Beggs HE, Baragona SC, Hemperly JJ, Maness PF (1997) NCAM140 interacts with the focal adhesion kinase p125(fak) and the SRC-related tyrosine kinase p59(fyn). J Biol Chem 272:8310-8319.

Black DL (2003) Mechanisms of alternative pre-messenger RNA splicing. Annu Rev Biochem 72:291-336.

Brand AH, Perrimon N (1993) Targeted gene expression as a means of altering cell fates and generating dominant phenotypes. Development 118:401-415.

Collins CA, Wairkar YP, Johnson SL, DiAntonio A (2006) Highwire restrains synaptic growth by attenuating a MAP kinase signal. Neuron 51:57-69.

Cremer H, Chazal G, Goridis C, Represa A (1997) NCAM is essential for axonal growth and fasciculation in the hippocampus. Mol Cell Neurosci 8:323-335.

Cunningham BA, Hemperly JJ, Murray BA, Prediger EA, Brackenbury R, Edelman GM (1987) Neural cell adhesion molecule: structure, immunoglobulin-like domains, cell surface modulation, and alternative RNA splicing. Science 236:799-806.

Dalva MB, McClelland AC, Kayser MS (2007) Cell adhesion molecules: signalling functions at the synapse. Nat Rev Neurosci 8:206-220.

Di Benedetto AJ, Klick Stoddard J, Glavan BJ (2001) Cloning and molecular characterization of a novel gene encoding a WD-repeat protein expressed in restricted areas of adult rat brain. Gene 271:21-31.

Dietzl G, Chen D, Schnorrer F, Su KC, Barinova Y, Fellner M, Gasser B, Kinsey K, Oppel S, Scheiblauer S, Couto A, Marra V, Keleman K, Dickson BJ (2007) A genome-wide transgenic RNAi library for conditional gene inactivation in Drosophila. Nature 448:151-156.

Ditlevsen DK, Kolkova K (2010) Signaling pathways involved in NCAMinduced neurite outgrowth. Adv Exp Med Biol 663:151-168.

Dubuque SH, Schachtner J, Nighorn AJ, Menon KP, Zinn K, Tolbert LP (2001) Immunolocalization of synaptotagmin for the study of synapses in the developing antennal lobe of Manduca sexta. J Comp Neurol 441:277-287.

Edenfeld G, Volohonsky G, Krukkert K, Naffin E, Lammel U, Grimm A, Engelen D, Reuveny A, Volk T, Klämbt C (2006) The splicing factor crooked neck associates with the RNA-binding protein HOW to control glial cell maturation in Drosophila. Neuron 52:969-980.

Fujita SC, Zipursky SL, Benzer S, Ferrús A, Shotwell SL (1982) Monoclonal antibodies against the Drosophila nervous system. Proc Natl Acad Sci U S A 79:7929-7933.

Grenningloh G, Rehm EJ, Goodman CS (1991) Genetic analysis of growth cone guidance in Drosophila: fasciclin II functions as a neuronal recognition molecule. Cell 67:45-57.

Groth AC, Fish M, Nusse R, Calos MP (2004) Construction of transgenic Drosophila by using the site-specific integrase from phage phiC31. Genetics 166:1775-1782.

Hartz BP, Ronn LC (2010) NCAM in long-term potentiation and learning. Adv Exp Med Biol 663:257-270.

Herold N, Will CL, Wolf E, Kastner B, Urlaub H, Lührmann R (2009) Conservation of the protein composition and electron microscopy structure of Drosophila melanogaster and human spliceosomal complexes. Mol Cell Biol 29:281-301.

Hortsch M, Bieber AJ, Patel NH, Goodman CS (1990) Differential splicing generates a nervous system-specific form of Drosophila neuroglian. Neuron 4:697-709.

Hummel T, Krukkert K, Roos J, Davis G, Klämbt C (2000) Drosophila Futsch/22C10 is a MAP1B-like protein required for dendritic and axonal development. Neuron 26:357-370.

Imlach W, McCabe BD (2009) Electrophysiological methods for recording synaptic potentials from the NMJ of Drosophila larvae. J Vis $\operatorname{Exp}(24)$ : 1109. doi:10.3791/1109.

Jan LY, Jan YN (1982) Antibodies to horseradish peroxidase as specific neuronal markers in Drosophila and in grasshopper embryos. Proc Natl Acad Sci U S A 79:2700-2704.

Johansen J, Halpern ME, Johansen KM, Keshishian H (1989) Stereotypic morphology of glutamatergic synapses on identified muscle cells of Drosophila larvae. J Neurosci 9:710-725.

Kittel RJ, Wichmann C, Rasse TM, Fouquet W, Schmidt M, Schmid A, Wagh DA, Pawlu C, Kellner RR, Willig KI, Hell SW, Buchner E, Heckmann M, Sigrist SJ (2006) Bruchpilot promotes active zone assembly, $\mathrm{Ca}^{2+}$ channel clustering, and vesicle release. Science 312:1051-1054.

Knittel LM, Copenhaver PF, Kent KS (2001) Remodeling of motor termi- 
nals during metamorphosis of the moth Manduca sexta: expression patterns of two distinct isoforms of Manduca fasciclin II. J Comp Neurol 434:69-85.

Koh YH, Popova E, Thomas U, Griffith LC, Budnik V (1999) Regulation of DLG localization at synapses by CaMKII-dependent phosphorylation. Cell 98:353-363.

Kosman D, Mizutani CM, Lemons D, Cox WG, McGinnis W, Bier E (2004) Multiplex detection of RNA expression in Drosophila embryos. Science 305:846.

Kristiansen LV, Hortsch M (2010) Fasciclin II: the NCAM ortholog in Drosophila melanogaster. Adv Exp Med Biol 663:387-401.

Lau CK, Bachorik JL, Dreyfuss G (2009) Gemin5-snRNA interaction reveals an RNA binding function for WD repeat domains. Nat Struct Mol Biol 16:486-491.

Leshchyns'ka I, Sytnyk V, Morrow JS, Schachner M (2003) Neural cell adhesion molecule (NCAM) association with PKCbeta2 via betaI spectrin is implicated in NCAM-mediated neurite outgrowth. J Cell Biol 161:625-639.

Lin DM, Goodman CS (1994) Ectopic and increased expression of Fasciclin II alters motoneuron growth cone guidance. Neuron 13:507-523.

Lin DM, Fetter RD, Kopczynski C, Grenningloh G, Goodman CS (1994) Genetic analysis of Fasciclin II in Drosophila: defasciculation, refasciculation, and altered fasciculation. Neuron 13:1055-1069.

Lisé MF, El-Husseini A (2006) The neuroligin and neurexin families: from structure to function at the synapse. Cell Mol Life Sci 63:1833-1849.

Littman DR, Thomas Y, Maddon PJ, Chess L, Axel R (1985) The isolation and sequence of the gene encoding T8: a molecule defining functional classes of T lymphocytes. Cell 40:237-246.

Lundquist EA, Herman RK (1994) The mec-8 gene of Caenorhabditis elegans affects muscle and sensory neuron function and interacts with three other genes: unc-52, smu-1 and smu-2. Genetics 138:83-101.

Mahr A, Aberle H (2006) The expression pattern of the Drosophila vesicular glutamate transporter: a marker protein for motoneurons and glutamatergic centers in the brain. Gene Expr Patterns 6:299-309.

Marie B, Sweeney ST, Poskanzer KE, Roos J, Kelly RB, Davis GW (2004) Dap160/intersectin scaffolds the periactive zone to achieve high-fidelity endocytosis and normal synaptic growth. Neuron 43:207-219.

Mayford M, Barzilai A, Keller F, Schacher S, Kandel ER (1992) Modulation of an NCAM-related adhesion molecule with long-term synaptic plasticity in Aplysia. Science 256:638-644.

McCabe BD, Hom S, Aberle H, Fetter RD, Marques G, Haerry TE, Wan H, O'Connor MB, Goodman CS, Haghighi AP (2004) Highwire regulates presynaptic BMP signaling essential for synaptic growth. Neuron 41:891-905.

Monani UR (2005) Spinal muscular atrophy: a deficiency in a ubiquitous protein; a motor neuron-specific disease. Neuron 48:885-896.

Muller D, Mendez P, Deroo M, Klauser P, Steen S, Poglia L (2010) Role of NCAM in spine dynamics and synaptogenesis. Adv Exp Med Biol 663: 245-256.

Muller D, Wang C, Skibo G, Toni N, Cremer H, Calaora V, Rougon G, Kiss JZ (1996) PSA-NCAM is required for activity-induced synaptic plasticity. Neuron 17:413-422.

Neubauer G, King A, Rappsilber J, Calvio C, Watson M, Ajuh P, Sleeman J, Lamond A, Mann M (1998) Mass spectrometry and EST-database searching allows characterization of the multi-protein spliceosome complex. Nat Genet 20:46-50.

Packard M, Mathew D, Budnik V (2003) FASt remodeling of synapses in Drosophila. Curr Opin Neurobiol 13:527-534.

Park Y, Rangel C, Reynolds MM, Caldwell MC, Johns M, Nayak M, Welsh CJ, McDermott S, Datta S (2003) Drosophila perlecan modulates FGF and hedgehog signals to activate neural stem cell division. Dev Biol 253:247-257.

Parnas D, Haghighi AP, Fetter RD, Kim SW, Goodman CS (2001) Regulation of postsynaptic structure and protein localization by the Rho-type guanine nucleotide exchange factor dPix. Neuron 32:415-424.

Persohn E, Pollerberg GE, Schachner M (1989) Immunoelectronmicroscopic localization of the $180 \mathrm{kD}$ component of the neural cell adhesion molecule N-CAM in postsynaptic membranes. J Comp Neurol 288:92-100.

Pollerberg GE, Schachner M, Davoust J (1986) Differentiation statedependent surface mobilities of two forms of the neural cell adhesion molecule. Nature 324:462-465.
Pollerberg EG, Sadoul R, Goridis C, Schachner M (1985) Selective expression of the $180-\mathrm{kD}$ component of the neural cell adhesion molecule N-CAM during development. J Cell Biol 101:1921-1929.

Pollerberg GE, Burridge K, Krebs KE, Goodman SR, Schachner M (1987) The $180-\mathrm{kD}$ component of the neural cell adhesion molecule N-CAM is involved in cell-cell contacts and cytoskeleton-membrane interactions. Cell Tissue Res 250:227-236.

Prokop A (2006) Organization of the efferent system and structure of neuromuscular junctions in Drosophila. Int Rev Neurobiol 75:71-90.

Rechsteiner M (1988) Regulation of enzyme levels by proteolysis: the role of pest regions. Adv Enzyme Regul 27:135-151.

Rørth P (1996) A modular misexpression screen in Drosophila detecting tissue-specific phenotypes. Proc Natl Acad Sci U S A 93:12418-12422.

Rubin GM, Hong L, Brokstein P, Evans-Holm M, Frise E, Stapleton M, Harvey DA (2000) A Drosophila complementary DNA resource. Science 287:2222-2224.

Schacher S, Wu F, Sun ZY, Wang D (2000) Cell-specific changes in expression of mRNAs encoding splice variants of aplysia cell adhesion molecule accompany long-term synaptic plasticity. J Neurobiol 45:152-161.

Schor IE, Rascovan N, Pelisch F, Alló M, Kornblihtt AR (2009) Neuronal cell depolarization induces intragenic chromatin modifications affecting NCAM alternative splicing. Proc Natl Acad Sci USA 106:4325-4330.

Schuster CM, Davis GW, Fetter RD, Goodman CS (1996a) Genetic dissection of structural and functional components of synaptic plasticity. I. Fasciclin II controls synaptic stabilization and growth. Neuron 17:641-654.

Schuster CM, Davis GW, Fetter RD, Goodman CS (1996b) Genetic dissection of structural and functional components of synaptic plasticity. II. Fasciclin II controls presynaptic structural plasticity. Neuron 17:655-667.

Sigrist SJ, Reiff DF, Thiel PR, Steinert JR, Schuster CM (2003) Experiencedependent strengthening of Drosophila neuromuscular junctions. J Neurosci 23:6546-6556.

Spartz AK, Herman RK, Shaw JE (2004) SMU-2 and SMU-1, Caenorhabditis elegans homologs of mammalian spliceosome-associated proteins RED and fSAP57, work together to affect splice site choice. Mol Cell Biol 24:6811-6823.

Spike CA, Shaw JE, Herman RK (2001) Analysis of smu-1, a gene that regulates the alternative splicing of unc-52 pre-mRNA in Caenorhabditis elegans. Mol Cell Biol 21:4985-4995.

Stapleton M, Carlson J, Brokstein P, Yu C, Champe M, George R, Guarin H, Kronmiller B, Pacleb J, Park S, Wan K, Rubin GM, Celniker SE (2002) A Drosophila full-length cDNA resource. Genome Biol 3:RESEARCH0080.

Stewart BA, Schuster CM, Goodman CS, Atwood HL (1996) Homeostasis of synaptic transmission in Drosophila with genetically altered nerve terminal morphology. J Neurosci 16:3877-3886.

Stewart BA, Atwood HL, Renger JJ, Wang J, Wu CF (1994) Improved stability of Drosophila larval neuromuscular preparations in haemolymphlike physiological solutions. J Comp Physiol A 175:179-191.

Sugaya K, Hongo E, Ishihara Y, Tsuji H (2006) The conserved role of Smul in splicing is characterized in its mammalian temperature-sensitive mutant. J Cell Sci 119:4944-4951.

Thibault ST, et al. (2004) A complementary transposon tool kit for Drosophila melanogaster using P and piggyBac. Nat Genet 36:283-287.

Tian M, Maniatis T (1993) A splicing enhancer complex controls alternative splicing of doublesex pre-mRNA. Cell 74:105-114.

Tsuda H, Han SM, Yang Y, Tong C, Lin YQ, Mohan K, Haueter C, Zoghbi A, Harati Y, Kwan J, Miller MA, Bellen HJ (2008) The amyotrophic lateral sclerosis 8 protein VAPB is cleaved, secreted, and acts as a ligand for Eph receptors. Cell 133:963-977.

Ule J, Jensen K, Mele A, Darnell RB (2005) CLIP: a method for identifying protein-RNA interaction sites in living cells. Methods 37:376-386.

Ullrich B, Ushkaryov YA, Südhof TC (1995) Cartography of neurexins: more than 1000 isoforms generated by alternative splicing and expressed in distinct subsets of neurons. Neuron 14:497-507.

Vactor DV, Sink H, Fambrough D, Tsoo R, Goodman CS (1993) Genes that control neuromuscular specificity in Drosophila. Cell 73: $1137-1153$ 
Wagh DA, Rasse TM, Asan E, Hofbauer A, Schwenkert I, Dürrbeck H, Buchner S, Dabauvalle MC, Schmidt M, Qin G, Wichmann C, Kittel R, Sigrist SJ, Buchner E (2006) Bruchpilot, a protein with homology to ELKS/ CAST, is required for structural integrity and function of synaptic active zones in Drosophila. Neuron 49:833-844.

Wodarz A, Hinz U, Engelbert M, Knust E (1995) Expression of crumbs confers apical character on plasma membrane domains of ectodermal epithelia of Drosophila. Cell 82:67-76.

Wright J, Copenhaver PF (2001) Cell type-specific expression of fasciclin II isoforms reveals neuronal-glial interactions during peripheral nerve growth. Dev Biol 234:24-41.

Wright JW, Snyder MA, Schwinof KM, Combes S, Copenhaver PF (1999) A role for fasciclin II in the guidance of neuronal migration. Development 126:3217-3228.
Zhou Z, Licklider LJ, Gygi SP, Reed R (2002) Comprehensive proteomic analysis of the human spliceosome. Nature 419:182-185.

Zinsmaier KE, Eberle KK, Buchner E, Walter N, Benzer S (1994) Paralysis and early death in cysteine string protein mutants of Drosophila. Science 263:977-980.

Zinsmaier KE, Hofbauer A, Heimbeck G, Pflugfelder GO, Buchner S, Buchner $E$ (1990) A cysteine-string protein is expressed in retina and brain of Drosophila. J Neurogenet 7:15-29.

Zito K, Fetter RD, Goodman CS, Isacoff EY (1997) Synaptic clustering of Fascilin II and Shaker: essential targeting sequences and role of Dlg. Neuron 19:1007-1016.

Zito K, Parnas D, Fetter RD, Isacoff EY, Goodman CS (1999) Watching a synapse grow: noninvasive confocal imaging of synaptic growth in Drosophila. Neuron 22:719-729. 